\title{
Free and Open Source Software for Computational Chemistry Education
}

Susi Lehtola, a) and Antti J. Karttunen ${ }^{2}$

1) Molecular Sciences Software Institute, Blacksburg, Virginia 24061, United States

${ }^{2)}$ Department of Chemistry and Materials Science, Aalto University, Espoo, Finland

Long in the making, computational chemistry for the masses [J. Chem. Educ. 1996, 73, 104] is finally here. Our brief review on various free and open source software (FOSS) quantum chemistry packages points out the existence of software offering a wide range of functionality, all the way from approximate semiempirical calculations with tight-binding density functional theory to sophisticated $a b$ initio wave function methods such as coupled-cluster theory, both for molecular and for solid-state systems. Combined with the remarkable increase in the computing power of personal devices, which now rivals that of the fastest supercomputers in the world of the 1990s, we demonstrate that a decentralized model for teaching computational chemistry is now possible thanks to FOSS computational chemistry packages, enabling students to perform reasonable modeling on their own computing devices, in the bring your own device (BYOD) scheme. FOSS software can be made trivially simple to install and keep up to date, eliminating the need for departmental support, and also enables comprehensive teaching strategies, as various algorithms' actual implementations can be used in teaching. We exemplify what kinds of calculations are feasible with four FOSS electronic structure programs, assuming only extremely modest computational resources, to illustrate how FOSS packages enable decentralized approaches to computational chemistry education within the BYOD scheme. FOSS also has further benefits: the open access to the source code of FOSS packages democratizes the science of computational chemistry, and FOSS packages can be used without limitation also beyond education, in academic and industrial applications, for example. For these reasons, we believe FOSS will become ever more pervasive in computational chemistry.

\section{CONTENTS}

\section{Introduction}

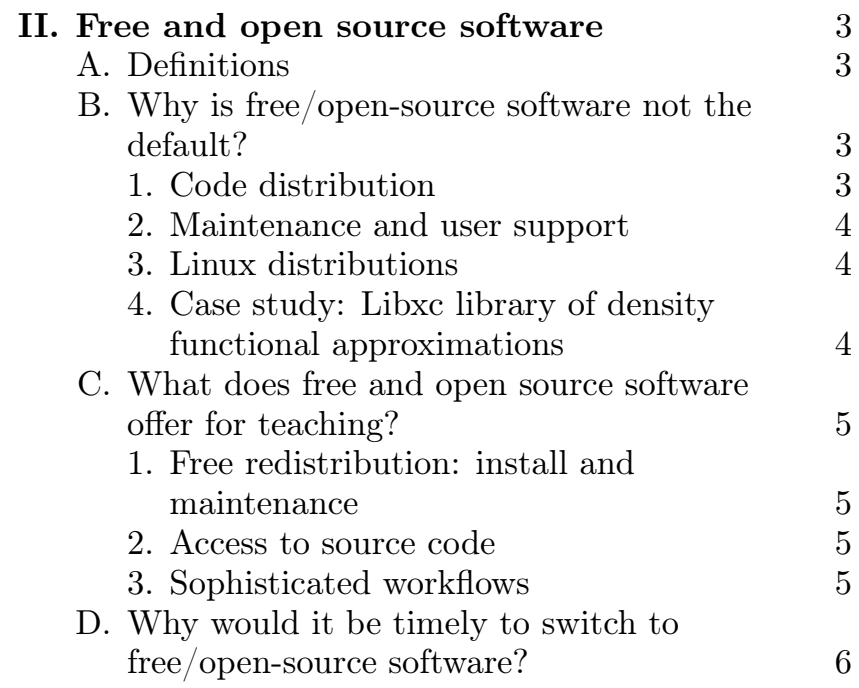

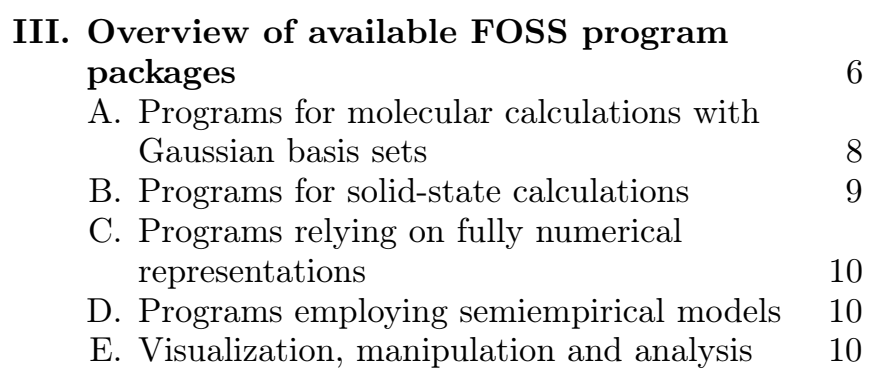

\footnotetext{
a)Electronic mail: susi.lehtola@alumni.helsinki.fi
}

\begin{tabular}{|c|}
\hline IV. Illustrations of feasible computations \\
\hline A. xtb \\
\hline B. NWChem \\
\hline C. Psi4 \\
\hline 1. Methylcyclohexane \\
\hline 2. Geometry of chromyl fluoride \\
\hline \begin{tabular}{|l|l} 
D. Quantum Espresso & \\
\end{tabular} \\
\hline 1. Optimal geometry \\
\hline 2. Band structure \\
\hline V. Summary and conclusions \\
\hline Supporting Information \\
\hline \\
\hline Acknowledgments \\
\hline References \\
\hline
\end{tabular}

\section{INTRODUCTION}

Quantum chemical research methods have been used extensively in the chemical industry already for several decades 1 [1 1 In addition to the widespread use in industry as well as in academia, quantum chemistry is also utilized in chemical education to provide atomic-level understanding of fundamental chemical concepts and phenomena $\sqrt[56]]{[6}$ For example, in undergraduate general and organic chemistry curricula, students get hands-on experience on concepts such as three-dimensional molecular structure, structural isomerism, conformers, and stereochemistry by means of computational exercises or computer laboratory sessions ${ }^{7}[9$

Although some of the aforementioned aspects can in principle be studied even with simpler methodolo- 
gies such as classical force fields, quantum chemical calculations with state-of-the-art software packages allow students to get firsthand understanding on more advanced topics such as molecular orbitals, chemical bonding, energetics, $\frac{10}{10}$ thermodynamics $\frac{11] 12}{112}$ reaction mechanisms, $\frac{13}{13}$ and various spectroscopies $\frac{14}{18}$

The ability to interpret and understand chemical phenonema with the help of quantum chemical calculations is a valuable skill in every chemist's professional life: nowadays, a significant portion of even the experimental studies reported in the chemical literature are tightly integrated with quantum chemical investigations. Moreover, as quantum chemistry is the critical bridging component between experimental work and machine learning methods, the ability to run quantum chemical calculations can be expected to become even more increasingly relevant and necessary to work-life in the near future.

Although computational chemistry for the massesa pervasive inclusion of computational modeling in the chemistry curriculum - has been long thought to be coming, $\frac{19}{19}$ it does not appear to have arrived yet. In their recent overview, ${ }^{20}$ Grushow and Reeves have summarized some select landmarks in computational chemistry education, noting at the same time how computational chemistry still has a somewhat limited presence in undergraduate curriculums, which can be attributed at least in part to the history of computational chemistry software.

In the 1990s, commercial software companies started selling graphical user interfaces to their quantum chemistry packages, some of which were particularly geared towards educational use. Such software was and still is typically used in a computer classroom setting, where a limited number of relatively powerful desktop computers are available for the students during the teaching sessions. The benefit of a computer classroom setting is that all software can be pre-installed for the students and the standardized software environment makes the possibilities (and limitations) of the software setup clear for the teachers in charge of the educational content. However, the computer classroom approach has limited scalability, as the number of students is limited by the number of workstations; this often makes the approach impractical for large-scale undergraduate teaching. Furthermore, while the computer classroom setting may be useful for teaching during contact sessions, the students' possibilities for running calculations outside the contact sessions are limited by the requirement of physical access to the computer classroom - which has proved to be challenging especially during the ongoing global coronavirus disease pandemic which has required social distancing. Lastly, the classroom setting typically limits the teacher and students to the pre-installed software, while costs for the required software licenses can be unfeasibly high for educational institutions with limited budgets; someone also has to maintain the software on the classroom computers and ensure it is kept up to date.

In the early 2000s, the WebMO package introduced a web-based approach to computational chemistry education, where the quantum chemistry software packages only need to be installed and maintained on a central server, and the teachers and students can then access the software through a web browser interface ${ }^{2122}$ A number of quantum chemistry software packages have been integrated with WebMO whose integrated molecular editor and analysis tools make it a rather low-barrier interface to quantum chemistry. As the users thus only need a web browser to access the computing software, WebMO was the first tool to enable a bring your own device (BYOD) paradigm in computational chemistry, in which the students can use their personal devices to take part in the teaching.

However, WebMO still requires someone to set up and administer the WebMO server, even though the need to purchase actual server hardware has been removed by the possibility of installing the service on cloud platforms such as the Amazon Web Services or the Google Cloud. Recently, the cloud-based Chem Compute platform has also begun to offer web access to computational chemistry software and computing resources for undergraduate teaching and research without any cost to the teachers,,$\underline{23}$ enabling such access for institutions that do not have the personnel or financial resources to set up their own physical or cloud servers; however, Chem Compute relies on computational resources volunteered by third parties whose continued future availability is not guaranteed.

As discussed above, great advances like WebMO and Chem Compute have been made in the direction of the BYOD paradigm, to which many universities have already shifted in order to cut down on the costs associated with the now-deprecated computer classroom model. In this work, we will show that free and open source software (FOSS) can be used in the context of the BYOD paradigm to achieve computational chemistry for the masses, all the while democratizing science by tearing down established power structures and barriers for research and education. (Inroads into BYOD in the context of virtual laboratories have also been recently discussed by Kobayashi et al. ${ }^{24}$ )

The layout of this work is as follows. In section II, we will begin by defining what we mean by FOSS (section II A). Then, we discuss why FOSS has not been the norm in science (section II B), what FOSS enables for the teaching of computational chemistry (section II C), and why it would be a good time now to switch over to FOSS in teaching (section IID). We present a brief overview of available FOSS packages in section III. We include several practical demonstrations of using state-of-the-art FOSS programs for computational chemistry education in section [IV] showcasing the kinds of calculations that are possible assuming only limited computer resources. The article concludes in a brief summary and discussion in section $\mathrm{V}$ 


\section{FREE AND OPEN SOURCE SOFTWARE}

\section{A. Definitions}

As some of the readers may not be familiar with the concept of FOSS, some definitions are necessary before the present discussion can take place. For the purposes of this article, we will adopt three key criteria for FOSS:

1. The ability of anyone to freely use the software for any purpose.

2. The ability to freely study the operation of the software, and modify it at will.

3. The ability to freely redistribute copies of the software - as well as modified versions thereof - to others.

Consequently, any software that does not satisfy these criteria for FOSS is referred to as proprietary or closed source software.

What is the significance of these criteria? The first criterion means simply that there can be no limitations on potential uses of the software: for instance, in addition to use in academic research and education, commercial use must also be permitted by the license. Moreover, the first criterion bars license terms that prohibit use of the software for purposes deemed questionable by the licensors, such as use in nuclear power plants or in research on genetic engineering. FOSS can be used by anyone for anything.

The second criterion means that the source code of the software must not only be available, but also that customizations to the source code must be allowed. This is of major importance for developing new features or computational models, for example. Being able to use software written by other authors to accomplish certain tasks eliminates the need to "reinvent the wheel" and thereby results in faster scientific development.25 This phenomenon has traditionally been the main enticement of contributing to closed-source or "open teamware, packages, as access to their source code partly eliminates the need to start from scratch, as algorithms implemented in the package by its other contributors can be leveraged to develop new computational models.

However, the control of access to the source code of such closed-source programs lead to perpetuating power structures and may inhibit academic collaborations between authors of different program packages, 27 instead of the Popperian ideal of science: the selfless pursuit of truth, 28 and a fair and unbiased competition of ideas and methods in the context of computational chemistry. Key persons in control of the access to the source codes of various software packages are able to hold back equitable competition and collaboration between scientists developing new methods and algorithms. The issue with gatekeepers is not a new phenomenon: as was already quipped by Max Planck, "A new scientific truth does not triumph by convincing its opponents and making them see the light, but rather because its opponents eventually die, and a new generation grows up that is familiar with it"; this apt observation is supported by a recent study that investigated the dynamics of scientific evolution with the standard empirical tools of applied

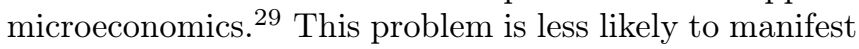
in FOSS, for the following reason.

The third criterion means that anyone who has a copy of the software can redistribute it to others. One does not need to ask case-by-case permission from the authors of the software in order to share it with one's collaborators or the reviewers of a scientific paper, for instance. It also means that anyone who has added new features to the program can freely distribute their version. This eliminates the problematic role of the gatekeepers in the "open teamware" model, as alternative versions of the software commonly known as forks can be distributed. It also eliminates the possibilities of the infamous practice 30 of preventing one's competitors from using one's software, which may have the result of hiding deficiencies and bugs in one's software. Case in point: the "war on supercooled water"31 exemplifies the problems of having prominent figures as exclusive gatekeepers. The "war" was only resolved once Princeton scientists gained access to their Berkeley competitors' source code and found a coarse error in the Berkeley source code ${ }^{32}$ Such problems are much less likely to exist if FOSS is used, as such programs are freely redistributable and can be thoroughly inspected by anyone.

In our opinion, the three criteria laid out above condense the essence of both the generally accepted 10-item definition for open source software by the Open Source Initiative ${ }^{33}$ as well as the four essential freedoms of "free software" or "libre software" defined by the Free Software Foundation ${ }^{34}$ Note that there is a wide variety of FOSS licenses that fit these criteria and that can be adopted by software projects, and that new software projects should choose their license with care $\stackrel{35}{\text { It }}$ is always easier to switch to a more permissible license later on than to move to a more restrictive license: any versions released under a FOSS license will continue being FOSS in the future, as well, even if newer versions switch to using a proprietary license, for example.

\section{B. Why is free/open-source software not the default?}

\section{Code distribution}

The ideology of FOSS is in line with the demands of science, ${ }^{36}$ as much like the Schrödinger or Dirac equation, computational models should ideally always be publicly available. Moreover, as the initial development and ongoing use of most scientific software has been and is funded by public research funding, the results of such work - the developed program source code - should be available to everyone. 
It is worthwhile to comment on the reasons for the longstanding status quo. As discussed by Hinsen 37 , before the advent of electronic computers, algorithms were developed with pen and paper, and the traditional paper journal article format is ideally suited to fully describe such algorithms. However, when implemented on a computer, algorithms often become too complicated to thoroughly describe in a journal article, and significant portions of the implementation are always left out. As this tacit information on what happens "under the hood" of various computational chemistry packages is typically passed only within the academic groups contributing to that code, lack of access to the source code creates another barrier of entry for third parties, and again ends up perpetuating established power structures.

However, nowadays there are well-established ways for distributing scientific software. Version control systems such as Git ${ }^{38}$ facilitate robust development of software, which can be hosted at no cost on sites such as GitHub 39 and GitLab ${ }^{40}$. GitHub and GitLab also enable a community approach to code development through the use of public code review, which is leveraged by many program packages to improve code quality and to decrease the learning curve for potential new contributors to the package. Stable releases of software can be made available on Open Science data repositories such as Zenodo $\underline{41}$ with version-specific Digital Object Identifiers (DOIs). Also precompiled versions can nowadays be easily distributed, as we will discuss in section III

\section{Maintenance and user support}

A commonly referred impediment of FOSS in science is that funding its maintenance and/or user support is challenging. ${ }^{26 / 42] 43}$ However, there are several companies whose whole business model is founded on the use and development of FOSS. For instance, Red Hat Inc broke $\$ 1$ billion in annual revenue in 2012, and its revenue has increased ever since, surpassing $\$ 3$ billion in 2018. ${ }^{44}$ There is clearly money to be made in selling support for FOSS. Moreover, at variance to proprietary software, maintenance and support for FOSS can be acquired from third parties if the original author(s) are either unavailable or unwilling to support for their code; this is the key to the Red Hat style business model.

As evidenced by forums such as the Computational Chemistry List 45 and the present authors' professional experience, online peer-to-peer user support-whose motivations have been studied e.g. by Constant, Sproull, and Kiesler ${ }^{46}$ is invaluable even in the case of proprietary programs. In the case of FOSS, this peer-to-peer support has an enhanced role, and is one of the keys behind the success of FOSS ${ }^{47}$ Because anyone can modify the software and distribute modified copies thereof, anyone can fix the bugs they run into, and gain fame even for small contributions. Also other aspects of the economic principles of FOSS have been studied extensively:
FOSS is a public good!490]62 Participation in the development and support of FOSS has been found to be more motivating than that of proprietary software, $\underline{6364}$ and participation in FOSS projects is motivating and carries economic benefits. $[5] 66$ FOSS promotes peer review, free exchange of ideas, and maintainability, ${ }^{67}$ and competition of FOSS packages promotes innovation. $\underline{68}$

\section{Linux distributions}

The Linux operating system is a prime example of FOSS. Originating from the University of Helsinki, it is nowadays ubiquitous. It is used in billions of mobile phones, laptops, workstations, as well as servers and compute clusters all around the world. All supercomputers on the TOP500 list ${ }^{69}$ and the majority of the world's internet servers have run on Linux for a long time; Android smartphones likewise run on Linux. Because of Linux, proprietary operating systems have been irrelevant in computational science for several decades. Chemists had good reasons to switch to Linux already ages ago, $\stackrel{70}{,}$ the present authors have used Linux as their main computational research platform for over 20 years.

A valuable feature of Linux distributions is that they are usually cross-platform: in addition to the usual x86 and x86-64 platforms, consisting of processors by e.g. the Intel Corporation and Advanced Micro Devices Inc. (AMD), Fedora packages are also available on s390x processors used on IBM mainframe computers and ARM processors such as the ones used in Raspberry Pi and new Mac computers, for instance. This versatility allows the use of heterogeneous hardware and ensures seamless compatibility even if students have dissimilar computing devices at their disposal.

Several Linux distributions, such as Ubuntu, Debian, and Fedora Linux have also solved the problem of efficient distribution of software many decades ago. Our criteria for FOSS in section IA allow such scientific software to be packaged as part of Linux distributions, and indeed several powerful program packages are already available as distribution packages thanks to the grand entrance of FOSS software in quantum chemistry in recent years. Some FOSS quantum chemistry packages like Erkale, ${ }^{71} \mathrm{Psi}_{4}{ }^{72}$ and its predecessor Psi373, and $\mathrm{PySCF}^{74}$ have been developed in a fully free/open-source development model since their beginning, while other packages that originated within a closed-source licensing model have also become open-sourced recently, such as OpenMolcas, ${ }^{75}$ Dalton, ${ }^{76}$ and NWChem. ${ }^{77}$

\section{Case study: Libxc library of density functional approximations}

An example of a successful scientific FOSS can be found in the Libxc library of density functional approximations. $\frac{78}{78}$ The modular library currently im- 
plements over 600 density functional approximations such as PBE, ${ }^{79} \mathrm{~B} 3 \mathrm{LYP}, 80$ and SCAN, 81 and is used by over 30 electronic structure programs ranging from programs using Gaussian basis sets (Erkale, ${ }^{71}$ Psi4, ${ }^{72}$, PySCF ${ }^{74}$ ) to plane-wave codes (Quantum Espresso ${ }^{82}$ ), finite element programs (HelFEM ${ }^{83} \sqrt[86]{ }, \mathrm{DFT}_{-\mathrm{FE}}{ }^{[87}$ ), and multiresolution adaptive grids (MADNESS ${ }^{88}$ ). In order to facilitate wider use by the community, Libxc recently switched to a more permissible FOSS license that allows Libxc to be more easily included in closedsource programs. Libxc is now used in several proprietary/commercial software packages, e.g. the Slatertype orbital ADF package ${ }^{89}$, and the Gaussian-type orbital GAMESS-US, ${ }^{90}$ Molpro, ${ }^{91}$ MRCC, ${ }^{92}$ ORCA,,$[3]$ and TURBOMOLE $^{94}$ programs; several other packages are also contemplating to migrate to Libxc.

The advantages of the community adoption of Libxc are manifold. A new density functional approximation only needs to be implemented in Libxc for it to become usable in any of the programs that use it, underlining the efficiency of the modular FOSS model. Moreover, access to the same implementation of a density functional approximation enables e.g. the study of reproducibility across various numerical approaches, which is important to be able to compare results obtained with different methods or software packages. Indeed, economic gains in terms of software development productivity and product quality can be achieved by reuse of mature FOSS components that are of the highest quality

We believe that computational chemistry will continue to transform by adopting more and more FOSS components, the Electronic Structure Library (ESL) is one of the notable pushes in this direction. ${ }^{97}$ Welldesigned, modular FOSS components can be maintained even by a single academic group; the semi-empirical dispersion library of the Grimme group is a successful recent example. 98

\section{What does free and open source software offer for teaching?}

\section{Free redistribution: install and maintenance}

In addition to its benefits for general use cases, 101 FOSS has three major advantages for teaching: the availability of the source code, the availability of precompiled binaries, as well as the general applicability of the software beyond academia. Starting out with the first advantage, software that satisfies the criteria for FOSS discussed in section II A can be redistributed, and included in Linux distributions, for example. This greatly facilitates the installation of these programs, as prepackaged software can be installed in a matter of minutes on a wide range of hardware, ranging from students' laptops to compute servers, simply by running a single command, or alternatively, finding the program in the distribution's graphical application manager and clicking on "Install".
We wish to note here that although installing scientific software by hand by compiling from source code affords customized tunings that may result in faster operation, that is, decreased runtimes of quantum chemistry packages, in many cases the gains realizable in computational chemistry education or small-scale computing are relatively modest and pale in comparison with the ease of effort afforded by the centralized packaging system: compiling from source takes a lot of time as well as expertise, and can lead to poor performance if the compiler options are not adequately chosen; note that several proprietary programs have likewise adopted a binary-only distribution model with the same limitations.

However, installation is only a part of the problem: the software must also be kept up to date. This does not happen automatically, and a constant level of administration effort is then required to monitor new releases, and to download and install new versions of the software. At variance, the Linux distribution packages get automatically updated with the rest of the system whenever new package versions come out: Linux package managers not only handle updates to the Linux operating system kernel, but also all other software, such as the internet browsers, the email clients, the office productivity software suites, the Fortran and $\mathrm{AT}_{\mathrm{EX}}$ compilers, and so on.

\section{Access to source code}

The second advantage of FOSS is that as the source code is available, it can be used in teaching. For instance, a course on electronic structure calculations can exemplify the basic algorithms by showing how they are implemented in an openly available program. Some codes go even further: for instance, Psi4Numpy ${ }^{102}$ is a project that aims to supply simple, easily modifiable Python algorithms for educational and proof-of-concept purposes. The PySCF quantum chemistry program 74 makes it easy to override and customize all algorithms, as they are mostly written in Python. Access to these kinds of projects not only facilitates research in and development of new electronic structure methods, but also means that teaching no longer has to be limited to pen and paper exercises but instead can also include real-life demonstrations. For example, an advanced course on electronic structure theory could involve asking students to write their own, customized solver for self-consistent field theory! 103

\section{Sophisticated workflows}

The third advantage of FOSS for teaching is that since students (like anyone else) can access the full power of various computational chemistry programs, they also have the the possibility to develop more general technical skills such as programming and interfacing programs with 
each other, for instance by generating sophisticated workflows that automate complex tasks. Automated workflows are highly useful tools for practical computations, as they can be leveraged to easily run and analyze thousands to even millions of calculations, which is needed for high-performance screening of materials, for instance. As was summarized in the first criterion in section IIA. FOSS software can also be freely used without limitations in industry to develop new thermoelectric energy conversion materials $\frac{104}{10}$ or semiconductor devices, $\stackrel{105}{10}$ for example, underlining its freedom and flexibility.

\section{Why would it be timely to switch to free/open-source software?}

We have argued above that FOSS has important ramifications for the reproducibility of science and also has several advantages for teaching. Although it is possible to switch from proprietary programs to FOSS within the traditional setup based on computer classrooms and/or central compute servers, there is yet another important aspect to consider: the BYOD approach discussed in section [1. In this section, we wish to examine FOSS from the point of view of the ongoing paradigm shift to the BYOD scheme.

As the price of laptop computers has dropped, many students now bring their own devices to the classroom. This paradigm shift has also affected university policies. As the demand for computer classrooms has decreased significantly as most students anyhow prefer using their own devices, universities may find it cheaper to just offer a laptop to all students. For instance, the Faculty of Science of the University of Helsinki, Finland pivoted to such an approach several years ago. As a result, the university has been able to cut down on computer classrooms that are expensive to maintain even while several students refuse the laptop offered by the university and opt to using their private laptops instead.

Although as was already discussed in section II a centralized compute server approach is compatible with the BYOD paradigm, the effortless availability of FOSS programs can be used to finally bring computational chemistry to the masses and thereby truly democratize science. As FOSS software packages can be made instantly available to everyone, the FOSS approach is ideally suited for personal devices in the BYOD approach. Such a distributed approach is optimal also for massive open online courses (MOOCs), as enrollment does not have to be limited based on the available centralized computer resources; instead, the students can run all of the necessary calculations on their own hardware.

Naturally, certain tradeoffs are implied in a course employing heterogeneous BYOD approaches, as one cannot assume personal devices to have the same computational power as purpose-built, dedicated compute servers. However, we argue that this is not much of an impediment due to the immense developments in the speed of processors and improved algorithms achieved during the past several decades. A concrete example of this is the TOP 500 list of supercomputers, which contains almost 30 years worth of data on the most powerful supercomputers in the world ${ }^{106 \mid 107}$ The estimated performance of the best and worst supercomputer on the list on a year-by-year basis is shown in figure 1 in units of $10^{9}$ floating-point operations per second (GFlops). Figure 1 also shows analogous benchmark data for commodity hardware: a cheap tablet computer with an Intel Celeron N4000 processor and a high-end business laptop with an Intel i7-10610U processor of one of the present authors (SL). A Raspberry $\mathrm{Pi} 4$ minicomputer was also assessed, and found to perform similarly to the Celeron N4000 processor.

As figure 1 illustrates, personal devices have performance in the tens to hundreds of gigaflops, which is comparable to the performance of fastest supercomputers of the mid 1990s, or to the slowest supercomputer on the TOP500 list in the mid 2000s. This amazing development in computational power means that the content of classic books on quantum chemistry such as Szabo-Ostlund 108 could be reproduced nowadays on commodity hardware; however, there's no reason to, since better computational methods and basis sets are available nowadays in many FOSS packages. Many calculations could probably be even carried out on an up-to-date smartphone!

The data in figure 1 suggest that a variety of calculations are possible within a reasonable time with personal devices. Combined with FOSS program packages that can be installed and kept up to date in a trivial fashion with a package manager, computational chemistry can finally be made available to the masses, as students are able to run (and modify!) FOSS packages on their own devices. The skills they gain doing so are directly transferable to both research and industry, as the same packages can also be used for heavy-duty calculations on supercomputers which is also freely allowed by their permissive license.

\section{OVERVIEW OF AVAILABLE FOSS PROGRAM PACKAGES}

This section presents an overview of available FOSS program packages for computational chemistry. As the number of FOSS projects has grown immensely in recent years, we restrict the overview to self-contained packages which are able to run quantum electronic structure calculations from atomistic input. FOSS for other types of molecular modelling has been discussed elsewhere, $109 \mid 110$ while various computational chemistry resources for education have been recently summarized by RodríguezBecerra et al. 111 .

As the availability of software is a moving goalpost, since new packages appear and old ones become technologically obsolete and stop being maintained, any review can by force of necessity only represent the situation at a given point in time. Continuously updated databases are 


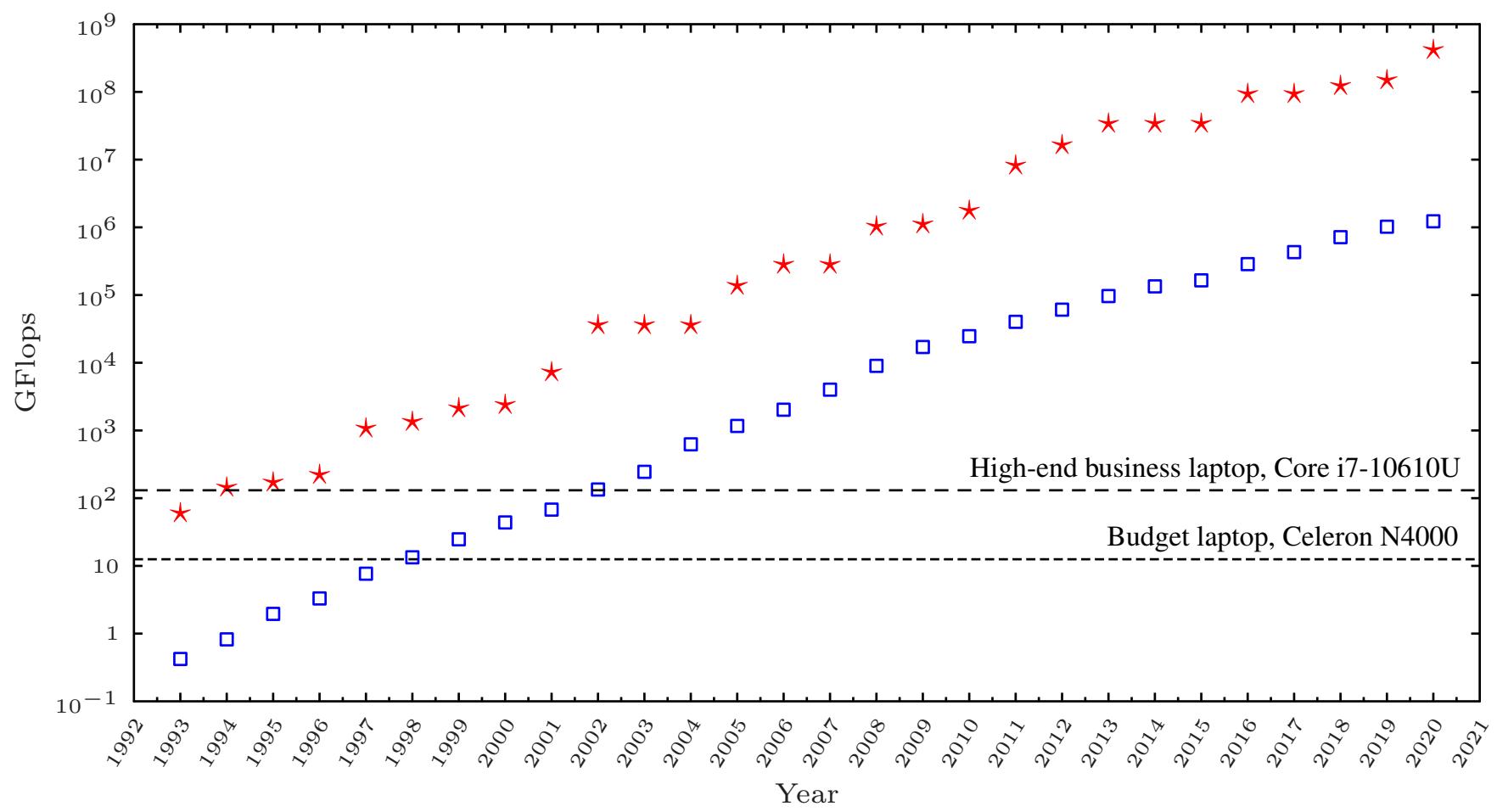

Figure 1. The best-performing (red stars) and worst-performing (blue squares) supercomputer on the TOP500 list $\frac{107}{10}$ as well as the performance of a budget laptop with a Celeron N4000 processor and a high-end business laptop with a Core i7-10610U processor (see supporting information). Note logaritmic scale on $y$ axis. The performance of Raspberry Pi 4 was found to be similar to Celeron N4000.

an alternative that is (hopefully) always up to date, 112 but any observations made on their basis similarly are tied to the time of observation and become outdated as enough time passes. For this reason, new reviews are typically published whenever the availability of software has changed enough.

The main goal of this section is merely to illustrate the breadth of software that is already available for use in computational chemistry. We have assembled the collection of packages by thorough internet searches. Because unmaintained packages are unlikely to be easy to install, or to become available as prepackaged software, we limit the overview to software that shows at least some development activity in recent years, as checked on the upstream development repositories. Even if it later turns out that we have missed some recently published software package in this review, or if some packages become replaced by newer competitors after the publication of this article, our main points should remain unaffected: there will likely still be a similar breadth of FOSS packages suitable for a variety of purposes within computational chemistry and computational chemistry education.

As FOSS, the programs listed here can be packaged and distributed openly without restriction; several of them are already available as part of Linux distributions such as Debian, Ubuntu, and Fedora Linux. Linux distribution packages are centrally maintained by the Linux distribution's packagers, and require no special knowl- edge or local department personnel to install them or keep the software up to date, at variance to typical proprietary packages. As we show in the Supporting Information, the packages can be installed on the command line; alternatively, they can also be installed using the distribution's application store. Importantly, the software is also automatically kept up to date by the distribution package manager, whereas the installation and upkeep of proprietary packages tends to require significant local expertise and time effort.

It is not even necessary to be running Linux to use such prepackaged programs. Windows users can run the software under the Windows Subsystem for Linux (WSL), which allows installing and using a Linux distribution easily inside Windows 10 . The cross-platform Python Package Index ${ }^{113}$ (PyPI) and Cond $2^{114}$ package managers are other alternatives for easy access to an increasing number of quantum chemistry packages both on Linux, Windows, and macOS. Computer laboratory settings can also be imitated using pre-made, customized live CDs or live USBs, for example.

Because of the large number of packages to review, we organize the discussion into

- programs for molecular calculations with Gaussian basis sets, section III A

- programs for solid-state calculations with various numerical approaches, section IIIB 
- programs employing fully numerical methods, section IIIC

- programs employing semiempirical methods, section IIID

- programs for visualization and manipulation, section IIIE

Due to space contraints, we only include minimalistic descriptions of the programs, and advise the reader to look up the programs' evolving capabilities in detail on the internet to assess their usefulness for a given computational chemistry course or other application. Most of the electronic structure programs support either Hartree-Fock (HF) and/or density functional theory 115|116 (DFT); several molecular programs also support various post-HF methods.

\section{A. Programs for molecular calculations with Gaussian basis sets}

Gaussian basis sets dominate the field of quantum chemistry, since all electrons can efficiently be included in the calculation, the electronic Coulomb integrals can be evaluated analytically in the Gaussian basis, 117 and the evaluation is efficient when recursion relations are used!118119 Thanks to many decades of work on the development of Gaussian basis sets, $120-122$ accurate basis sets exist for the accurate reproduction of various molecular properties at several levels of theory. Access to analytical integrals greatly facilitates the implementation of post-HF theories, and also guarantees accurate force and Hessian evaluations.

Bage ${ }^{123}$ : is a $\mathrm{C}++$ program package that features e.g. analytical CASPT2 [complete active space perturbation theory at the second order] nuclear energy gradients and derivative couplings, relativistic multireference wave functions based on the Dirac equation, and implementations of novel electronic structure theories.

Chronus Quantum ${ }^{124}$ : is a $\mathrm{C}++$ program package that focuses on the consistent treatment of time dependence and spin in the electronic wave function, as well as the inclusion of relativistic effects in said treatments.

Dalton 125: is a Fortran program that specializes in molecular properties at various levels of theory, such as frequency-dependent response properties; one-, two-, and three-photon processes, etc. In addition to $\mathrm{HF}$ and DFT, Dalton features several post-HF methods like multiconfigurational selfconsistent field (MCSCF) theory and coupledcluster theory.

Ergo ${ }^{126}$ : is a $\mathrm{C}++$ program for linear-scaling $\mathrm{HF}$ and DFT calculations for molecules.
ERKALE ${ }^{71}$ : is a $\mathrm{C}++$ program implementing $\mathrm{HF}$ and DFT that specializes in the modeling of inelastic $\mathrm{x}-$ ray spectroscopies, self-interaction corrected DFT, as well as various orbital localization methods.

$e^{T \text { 127: }}$ is a $\mathrm{C}++$ program primarily aimed for coupledcluster calculations of molecular systems, which specializes in multiscale and multilevel methods, as well as modern Cholesky decomposition techniques for two-electron integrals.

JuliaChem ${ }^{128}$ : is a Julia package for HF calculations.

LSDalton 125: is a Fortran code targeted for linearscaling HF and DFT calculations on large molecular systems, and also includes some coupled-cluster capabilities.

MolGW ${ }^{129}$ : is a Fortran $/ \mathrm{C}++$ package that implements HF and DFT, but specializes in many-body perturbation theory: the $G W$ approximation and the Bethe-Salpeter equation.

MPQC130: is a $\mathrm{C}++$ program for massively parallel quantum chemistry, which originally focused on $\mathrm{HF}$ and DFT but has later evolved support for post-HF many-body theories.

NWChem ${ }^{77}$ : is a major quantum chemistry package written in Fortran and has a variety of features for both molecular and solid-state calculations.

Psi4 ${ }^{72}$ : is a modular $\mathrm{C}++/$ Python package for HF, DFT and various post-HF calculations that can be used either as a traditional quantum chemistry package with simple and intuitive input files, or as Python modules for running calculations in Python.

PySCF 74: is a collection of Python modules for electronic structure calculations with significant capabilities also for solid-state simulations, including e.g. coupled-cluster implementations for crystalline systems.

PyQuante ${ }^{131}$ : is a Python package for quantum chemistry with some $\mathrm{C}$ extensions that emphasizes ease of understanding the code over performance.

OpenMolcas ${ }^{75}$ : is a Fortran package that specializes in multiconfigurational approaches to electronic structure theory, but also implements various DFT calculations, for example.

Serenity ${ }^{132}$ : is a $\mathrm{C}++$ program for subsystem quantum chemical methods.

SlowQuant ${ }^{133}$ : is a Python program for molecular quantum chemistry that derives its name from the use of Python for even the computational demanding parts of the program. 
VeloxChem ${ }^{134}$ : is a $\mathrm{C}++/$ Python package for molecular properties and for modeling various spectroscopies based on response theory.

Uquantchem 135 : is a Fortran 90 program written for HF, DFT, Møller-Plesset perturbation theory, configuration interaction singles and doubles, quantum Monte Carlo, etc.

\section{B. Programs for solid-state calculations}

The major difference between solid-state and molecular calculations is that the orbitals experience exponential decay in molecular calculations, while solid-state calculations are performed on periodic crystals where the wave function has to obey Bloch's theorem ${ }^{136}$ Because of the periodicity, calculations in the solid state are in many ways more difficult than those in molecules due to the need of k-point sampling, for instance; see ref. 137 for a recent introduction. Post-HF methods are much less prominent in the solid state than in molecules. Instead, calculations on solids are typically carried out with DFT

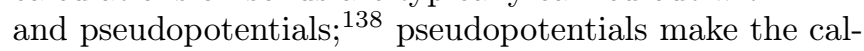
culations less costly while introducing an error which is typically negligible compared to the error in the density functional approximation itself.

The conventional way to model crystalline systems is to use plane waves. However, many other numerical schemes have also been pursued. Note that the programs listed here that employ (pseudo)atomic basis functions can naturally handle periodicity in $0,1,2$, or 3 dimensions, corresponding to atoms and molecules, chains, sheets, and crystals, respectively. Still, we have listed them as solid state codes because they are most often used for calculations with DFT and pseudopotentials.

ABINIT ${ }^{139}$ : is Fortran program for plane wave calculations that supports DFT as well as more advanced formalisms like many-body perturbation theory.

ACE-Molecule ${ }^{140}$ : is a $\mathrm{C}++$ program that employs uniform real-space grids of Lagrange sinc functions and pseudopotentials, and supports density functional calculations on both periodic and nonperiodic systems and wave function theory calculations based on Kohn-Sham orbitals.

BigDFT 141: is a Fortran program that is based on the use of pseudopotentials and a two-tier Daubechies wavelet basis to achieve a spatially localized basis.

Conquest $^{142}$ : is a Fortran program for large-scale DFT calculations employing pseudo-atomic orbital basis sets.

CP2K $^{143}$ : is a Fortran package based on Gaussian basis sets specializing in solid state physics, implementing HF, DFT, Møller-Plesset perturbation theory and the random phase approximation.
DFTK.j 144: or the density-functional toolkit is a collection of Julia routines for experimenting with planewave DFT that emphasises simplicity and flexibility.

ELK $^{145}$, EXCITING ${ }^{146}$, and FLEUR ${ }^{147}$ : are Fortran programs for linearised augmented-plane wave calculations which can reach microhartree accurate total energies for carefully chosen basis sets.

GPAW ${ }^{148}$ : is Python/C electronic structure program for DFT calculations within the projectoraugmented wave approach which supports three modes of operation: (i) finite-difference grids, (ii) numerical atomic orbitals, and (iii) plane waves.

JDFTX ${ }^{149}$ : is a $\mathrm{C}++$ plane wave DFT code aimed to be easy to develop and easy to use, whose key feature is support for joint DFT for the description of electronic systems in contact with molecular liquids.

M-SPARO150: is a MATLAB package for prototyping DFT calculations employing finite-difference grids and pseudopotentials.

Octopus 151: is a Fortran program based on pseudopotentials and finite difference grids that focuses on time-dependent DFT for handling non-equilibrium phenomena.

OpenMX $^{152}$ : is a C package for DFT calculations with pseudopotentials and numerical atomic orbitals.

PARSEC 153: is a Fortran program based on finitedifference grids for density functional calculations with pseudopotentials.

PWDFT.j 154: is a Julia package written from scratch to facilitate development of novel computational methods using plane waves.

$\mathbf{R M G}^{155}$ : is a $\mathrm{C}++$ /Fortran program employing real space grids and multigrid algorithms for density functional calculations with pseudopotentials.

Siesta 156: is a Fortran program for electronic structure calculations and ab initio molecular dynamics of molecules and solids that employs a basis set of numerical atomic orbitals, which are strictly localized, enabling the use of sparsity.

Qbox ${ }^{157}$ : is a $\mathrm{C}++$ program aimed for first principles molecular simulations using plane waves and pseudopotentials.

Quantum Espresso ${ }^{82}$ : is a Fortran/C program for plane wave calculations with pseudopotentials on a wide range of hardware from laptops to supercomputers.

SPARC158: is a C program for parallel DFT calculations employing finite-difference grids and pseudopotentials. 


\section{Programs relying on fully numerical representations}

The idea in modern fully numerical methods is to represent the orbitals directly in real space, and to use a representation of non-uniform accuracy (more grid points near the nuclei and fewer points in empty regions of the system) so that all-electron calculations become feasible. Although fully numerical approaches have a long history for calculations on atoms and diatomic molecules, 159 they are otherwise a relatively recent development in electronic structure theory and have only recently become competitive with e.g. Gaussian-basis calculations whenever high accuracy is needed $\underline{160}$

DFT-FE ${ }^{87}$ : is a $\mathrm{C}++$ program that employs spectral finite-element basis sets for a local real-space variational formulation of DFT, and is able to handle pseudopotential and all-electron calculations within the same framework and arbitrary periodicity.

HelFEM: is a $\mathrm{C}++$ program for fully numerical calculations on atoms 8486 and diatomic molecules 83 at the HF or DFT levels of theory employing highorder numerical basis functions and yielding fully variational energies.

MADNESS ${ }^{161}$ : is a $\mathrm{C}++$ program that relies on the use of multiresolution adaptive grids, which has been used in a variety of studies on novel real-space approaches to electron correlation, for instance.

MRChem 160 : is a $\mathrm{C}++$ program that also relies on multiresolution adaptive grids for Hartree-Fock and density functional calculations of molecules; its specialty is the computation of magnetic properties such as nuclear magnetic shielding constants.

$\mathbf{x} 2 \mathbf{d h} \mathbf{1 6 2}^{162}$ : is a Fortran program for non-relativistic finite difference restricted open-shell Hartree-Fock and density functional calculations on diatomic molecules.

\section{Programs employing semiempirical models}

Semiempirical models offer affordable techniques for approximate quantum mechanical calculations that fall in accuracy in-between $a b$ initio density functional calculations and force field techniques. Tight-binding $\mathrm{DFT}^{163}$ is probably the best-known semiempirical model, and it is available in several program packages. Other types of semiempirical methods exist as well, please refer to Thiel ${ }^{166}$ and Bannwarth et al. $\frac{167}{16}$ for discussion.

DFTB +168 : is a Fortran package for various calculations based on tight-binding DFT.

Latte $^{169}$ : is a Fortran program for tight-binding DFT molecular dynamics.
Sparrow 170: is a $\mathrm{C}++/$ Python program for fast semiempirical quantum chemical calculations, including tight-binding DFT.

$\mathbf{x t b}^{167}$ : is a Fortran package that implements various semiempirical eXtended Tight-Binding methods.

\section{E. Visualization, manipulation and analysis}

In addition to the actual computational chemistry codes, simplified frontends are often invaluable for initializing, visualizing and analyzing calculations. Several FOSS packages with graphical user interfaces are also available for this purpose; some even come with integration with FOSS electronic structure programs that allow running calculations within a graphical interface.

For creating models and visualizing computational results, FOSS graphical user interfaces such as Jmol171, Avogadro ${ }^{172}$, IQmol ${ }^{173}$ and PyMol14 can be installed and used. The Atomic Simulation Environment (ASE) ${ }^{175}$ contains versatile tools for building molecular and periodic models and enables easy retrieval of molecular structures from structural databases such as PubChem!176 The interconversion of various input and output file formats between different programs can be carried out for example with the Open Babe 177 and cclib $^{178}$ packages. Postprocessing of calculations can be carried out with e.g. the Multiwfn ${ }^{179}$ and ORBKIT ${ }^{180}$ packages.

\section{ILLUSTRATIONS OF FEASIBLE COMPUTATIONS}

To enable a practical demonstration of the BYOD paradigm within computational chemistry education, it is time to illustrate the easy access to several powerful FOSS quantum chemistry packages in two widely used Linux distributions, Fedora and Ubuntu. The Supporting Information contains practical step-by-step examples of combining the BYOD paradigm with FOSS packages to run quantum chemical calculations according to the BYOD-FOSS paradigm. Four different program packages are used in the practical illustrations: xtb (section IV A), NWChem (section IV B), Psi4 (section IV C), and Quantum Espresso (section IVD). Installation instructions are provided for each code and all examples can be run under Linux, macOS, or the Windows Subsystem for Linux. In all cases, the software can be installed in a matter of minutes on a personal computer, either using a Linux distribution package manager or the Conda package manager. For convenience, the supporting information is also available as a git repository! 181 


\section{A. $x t b$}

The primary design goal of xtb has been the fast calculation of structures and noncovalent interaction energies for molecular systems with up to roughly 1000 atoms. $167 \mid 182$ The GFN $n$-xTB methods implemented in $\mathrm{xtb}$ are semiempirical quantum chemical methods 167 parametrized for the whole periodic table up to radon $(Z=86)$. A highly attractive feature of $x t b$ is its performance: calculations on small molecules (10-20 atoms) finish in matter of seconds even on a low-powered laptop computer. xtb is a powerful tool for instance in the preoptimization of geometries and molecular conformations before computationally more demanding calculations; see ref. 183 for a recent application to water oxidation catalysis, for example.

The Supporting Information includes step-by-step guidelines for installing xtb and using it to study structures, conformations, energetics, and molecular orbitals of inorganic and organic molecules. Calculations on pharmaceutically relevant cisplatin and transplatin molecules shown in figure 2 are briefly summarized here to showcase the basic use of xtb. Cisplatin, cis- $\left[\mathrm{Pt}\left(\mathrm{NH}_{3}\right)_{2} \mathrm{Cl}_{2}\right]$, is a chemotherapy medication used in cancer treatments whose stereoisomer, transplatin, trans- $\left[\mathrm{Pt}\left(\mathrm{NH}_{3}\right)_{2} \mathrm{Cl}_{2}\right]$, is ineffective in cancer treatment.

The Pt(II) atom is square-planar coordinated in both cisplatin and transplatin. Which configuration, cis or trans, is lower in energy? We use the xtb program to answer this question. The first task is to have initial geometries for the two molecules. In general, initial geometries can be obtained from structural databases such as Pubchem, $\frac{176}{b u i l t ~ i n ~ a ~ g r a p h i c a l ~ u s e r ~ i n t e r f a c e ~ w i t h ~}$ programs such as Jmol, Avogadro, or IQMol; or built by hand in internal coordinates (bond lengths, angles and dihedrals) in the Z-matrix formalism, for example. Handbuilt molecular geometries for cisplatin and transplatin are given in XYZ format in figures 3 and 4 , respectively. While these geometries should be sufficiently close to optimal to allow for a straightforward optimization without difficulties, they are still quite rough in that the total energy is expected to change by several millihartrees in the geometry optimization, corresponding to changes in the energy of several $\mathrm{kcal} / \mathrm{mol}$.

The next step is to bring both molecules into a (local) minimum of the potential energy surface (PES) by optimizing the geometries with xtb. The point groups of the initial geometries are approximately $C_{2 v}$ and $C_{2 h}$ for cisplatin and transplatin, respectively, but symmetry is not enforced during the xtb optimizations. The only input needed by $x t b$ in this case are the cartesian coordinates of both molecules in XYZ format, which were given in figures 3 and 4 for cisplatin and transplatin, respectively.

The geometry optimizations complete in seconds even on a low-powered computer; the supporting information (SI) contains all of the necessary inputs. For cisplatin, the optimized $\mathrm{Pt}-\mathrm{Cl}$ and $\mathrm{Pt}-\mathrm{N}$ distances are $2.24 \AA$ and $2.15 \AA$, respectively. Considering the relatively low level of theory, the obtained distances are in reasonable agreement with the $\mathrm{Pt}-\mathrm{Cl}$ and $\mathrm{Pt}-\mathrm{N}$ distances of $2.25 \AA$ and $2.06 \AA$, respectively, obtained with the much higher-level methods of Tasinato, Puzzarini, and Barone ${ }^{184}$ who employed coupled-cluster theory with full single and double substitutions and perturbative triple substitutions, $\operatorname{CCSD}(\mathrm{T})$.

Comparing the total energies of the two stereoisomers after geometry optimization shows that the total energy of transplatin is $20 \mathrm{~kJ} / \mathrm{mol}$ lower, that is, more negative than that of cisplatin. This means that transplatin is the energetically more favorable stereoisomer of diamminedichloroplatinum(II), $\left[\mathrm{Pt}\left(\mathrm{NH}_{3}\right)_{2} \mathrm{Cl}_{2}\right]$. For comparison, Liu and Franke ${ }^{185}$ reported an energy difference of $56 \mathrm{~kJ} / \mathrm{mol}$ with a much higher level of theory: relativistic CCSD $(\mathrm{T})$ employing direct perturbation theory, a 13s9p7d5f2g contracted Gaussian basis for Pt and augcc-pVQZ for other elements, evaluated on top of molecular geometries optimized for the Becke'88-Perdew'86 functional. 186/187 The result from xtb, which we were able to get in a matter of seconds, is in good qualitative (or even semiquantitative!) agreement with the result obtained with the high level of theory. Next, in section IVB, we will revisit cisplatin and transplatin with DFT calculations that afford a step up in accuracy over $\mathrm{xtb}$.

\section{B. NWChem}

NWChem is a program that has been developed for almost 30 years. Consequently, a large number of features are available in the code: $\mathrm{HF}, \mathrm{DFT}$, as well as post-HF calculations, ab initio molecular dynamics, and so on. NWChem has been designed to run on high-performance parallel supercomputers as well as on conventional workstations. The Supporting Information includes step-bystep guidelines for installing NWChem and using it to study the same pharmaceutically relevant cisplatin and transplatin molecules that were studied with xtb in section IVA.

We choose to use non-empirical DFT in the NWChem examples. Although NWChem also includes more accurate $a b$ initio methods such as coupled-cluster theories, we shall not consider them in this work since their proper use requires much more understanding and computational power than DFT does, and as such methods are typically not included in undergraduate level courses. We choose the non-empirical PBE0 hybrid functiona $\sqrt{79|188| 189}$ (sometimes also known as hybrid PBE or PBEh) that provides reasonable geometries and energetics across the periodic table and shows good performance for complexes with $d$-and $f$-metals.190 191

Even though DFT is simpler than many post-HF theories, setting up adequate DFT calculations still requires some considerations. The one-electron basis set is one of the most important aspects to consider in any electronic structure calculation in general, such as our at- 

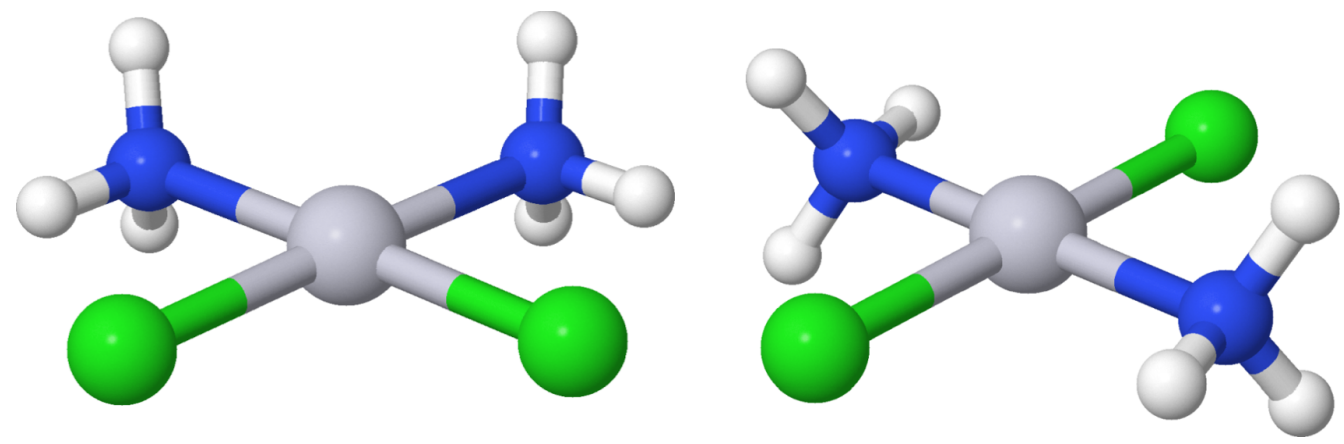

Figure 2. Cisplatin (left) and transplatin (right). Color coding: $\mathrm{Pt}=$ gray, $\mathrm{Cl}=$ green, $\mathrm{N}=$ blue, and $\mathrm{H}=$ white.

$\begin{array}{lrrr}11 & & & \\ \text { cis- [Pt (NH3) 2Cl2] } & \text { (cisplatin); } & \text { angstrom units } & \\ \text { Pt } & 0.00000000 & -0.00000000 & -0.19134710 \\ \text { Cl } & 0.00000000 & 1.61220407 & 1.42085566 \\ \text { Cl } & 0.00000000 & -1.61220407 & 1.42085566 \\ \text { N } & 0.00000000 & 1.40714181 & -1.59849021 \\ \text { H } & 0.81649658 & 1.30951047 & -2.16752575 \\ \text { H } & -0.81649658 & 1.30951047 & -2.16752575 \\ \text { N } & 0.00000000 & -1.40714181 & -1.59849021 \\ \text { H } & -0.81649658 & -1.30951047 & -2.16752575 \\ \text { H } & 0.81649658 & -1.30951047 & -2.16752575 \\ \text { H } & 0.00000000 & 2.30951093 & -1.16752621 \\ \text { H } & 0.00000000 & -2.30951093 & -1.16752621\end{array}$

Figure 3. Molecular geometry of cisplatin in XYZ format.

tempted PBE0 calculation with NWChem. The choice of the one-electron basis set has an immense importance on the computational cost and accuracy of the resulting calculations. While the GFN $n$-xTB methods discussed above in section IV A did not require the specification of a basis set, as the basis set is already an essential part of the specification of the GFN $n$-xTB methods themselves, the basis set - which parametrizes the allowed degrees of freedom for the movement of the electrons - does need to be specified for HF, DFT and post-HF calculations.

Because of the profound importance of the choice of the basis set, various types of Gaussian basis sets have a long history in quantum chemistry ${ }^{120}$ Although many readers will be familiar with traditional basis sets like STO-3G, ${ }^{192} 3-21 \mathrm{G}^{193}$ and $6-31 \mathrm{G}^{*},{ }^{194}$ the development of computer processors and quantum chemical models in recent decades have also lead to significant advances in basis set design. Hundreds of Gaussian basis sets intended for various purposes are nowadays available on the Basis Set Exchange $\frac{195}{195}$ for example.

Because the basis set is an approximation, it is highly desirable to be able to control its accuracy in order to make tradeoffs between the cost of the calculation and the accuracy of the obtained results. Accordingly, modern basis sets typically come in families of varying size. $\frac{1211122}{1.2}$ the smallest sets enable quick but qualitative calculations, while the larger sets enable quantitative computations at the cost of more computer time. In contrast to traditional basis sets, modern basis set families allow for a cost-efficient approach to the complete basis set limit, at which point the error in the one-electron basis set no longer affects the calculation. Note that also other types of basis sets than Gaussians may be used for quantum chemistry, see ref. 159 for further discussion.

In this work, we will only consider the Karlsruhe def2 family of Gaussian basis sets, ${ }^{196}$ which are a good allround choice for general chemistry as they are available for the whole periodic table up to radon $(Z=86)$. As radon is an element of the $6^{\text {th }}$ period, while relativistic effects are already essential for chemistry of the $5^{\text {th }}$ row, 197/198 relativistic effects are described in the def2 basis sets through the use of effective core potentials (ECPs) ${ }^{199}$ The ECP is used to describe the chemically inactive, deep-core electrons only implicitly; this also decreases the overall cost of the calculation.

The Karlsruhe def2 sets come at three levels of accuracy. Split-valence (SV) basis sets are the smallest reasonable basis set for general applications. The def2SVP basis is a SV basis set with polarization (P) functions, and is similar in size to the $6-31 \mathrm{G}^{* *}$ also known as the $6-31 \mathrm{G}(\mathrm{d}, \mathrm{p})$ basis set. Like $6-31 \mathrm{G}^{* *}$, the def2SVP set can also be used without polarization functions on hydrogen atoms; this basis is called def2-SV(P), it is smaller than the $6-31 \mathrm{G}^{*}$ basis, and it is often useful for quick qualitative/semi-quantitative calculations. For more quantitative calculations, the def2 series also 


\begin{tabular}{lrrr}
11 & & \\
trans- $[\mathrm{Pt}(\mathrm{NH})$ 2Cl2] & \multicolumn{3}{c}{ (transplatin) angstrom units } \\
Pt & 0.00000000 & 0.00000000 & 0.00000000 \\
$\mathrm{Cl}$ & 2.27999997 & -0.00036653 & 0.00000000 \\
$\mathrm{Cl}$ & -2.27999997 & 0.00036653 & 0.00000000 \\
$\mathrm{~N}$ & -0.00031991 & -1.98999997 & 0.00000000 \\
$\mathrm{H}$ & 0.46944690 & -2.32340883 & -0.81740913 \\
$\mathrm{H}$ & 0.46944690 & -2.32340883 & 0.81740913 \\
$\mathrm{~N}$ & 0.00031991 & 1.98999997 & 0.00000000 \\
$\mathrm{H}$ & -0.46944690 & 2.32340883 & -0.81740913 \\
$\mathrm{H}$ & -0.46944690 & 2.32340883 & 0.81740913 \\
$\mathrm{H}$ & 0.94318252 & 2.32318174 & 0.00000000 \\
$\mathrm{H}$ & -0.94318252 & -2.32318174 & 0.00000000
\end{tabular}

Figure 4. Molecular geometry of transplatin in XYZ format.

contains a triple- $\zeta$ valence polarization set (def2-TZVP) as well as a quadruple- $\zeta$ valence polarization set (def2QZVP), which typically suffice for achieving the complete basis set limit in HF and DFT calculations. Calculations at post-HF levels of theory, however, require larger basis sets with additional polarization functions; the def2TZVPP and def2-QZVPP basis sets exist for this purpose. Diffuse functions (D) are necessary for the proper description of anions as well as to model e.g. electric polarizabilities; sets are likewise available at all levels of accuracy (def2-SVPD, def2-TZVPD, def2-TZVPPD, def2-QZVPD, def2-QZVPPD) for this purpose.200

For the present demonstration, we choose the def2TZVP basis set, as triple- $\zeta$ basis sets are well-known to yield energies that are sufficiently close to the complete basis set limit (see also the applications in subsections IVC1 and IV C2. Although hybrid functionals are computationally more demanding than nonhybrid functionals, it is notable that the dispersioncorrected hybrid PBE0-D4 generalized gradient approximation (GGA) functional was recently shown to outperform the dispersion-corrected, meta-GGA-type nonhybrid $r^{2}$ SCAN-D4 functional in accuracy even for reaction energies of metal-organic reactions. 201

Having completed our introduction to DFT calculations, basis sets, and NWChem, similarly to the workflow in the case of xtb, the first task is to bring both molecules into a (local) minimum of the potential energy surface (PES) by means of geometry optimization. The geometry optimization is started from the same hand-built initial geometries presented in section IV A. In contrast to xtb, NWChem is capable of employing the point group symmetry $\left(C_{2 v}\right.$ and $C_{2 h}$ for cisplatin and transplatin, respectively) during the geometry optimization in order to speed up both the electronic structure calculation as well as the geometry optimization, and will do so by default. This means that the calculation runs faster, but also that the molecule is constrained to the same point group as the initial geometry during the whole optimization. If the user is not careful, this may also be a bad thing, as the use of symmetry may sometimes lead to convergence to a saddle point instead of a local minimum.
The input required for NWChem is more complicated than that for xtb. Running NWChem requires setting up an input file that contains various computational parameters in addition to the input geometry. Fully annotated input files can be found in the SI, a shortened example is shown in figure 5 .

The geometry optimizations of cisplatin and transplatin finish in a matter of minutes on one processor core, depending on the used computer. The optimized $\mathrm{Pt}-\mathrm{Cl}$ and $\mathrm{Pt}-\mathrm{N}$ distances for cisplatin are $2.28 \AA$ and $2.08 \AA$, respectively. These values are in excellent agreement with the values of Tasinato, Puzzarini, and Barone ${ }^{202}$ that were discussed in section IV A, that is, $\mathrm{Pt}-\mathrm{Cl}$ and $\mathrm{Pt}-\mathrm{N}$ distances of $2.25 \AA$ and $2.06 \AA$, respectively: the geometries agree to $0.03 \AA$.

Next, comparing the total PBE0/def2-TZVP energies of the two stereoisomers shows that transplatin is $54 \mathrm{~kJ} /$ mol lower (more negative) than cisplatin. Our DFT value is in good quantitative agreement with the energy difference of $56 \mathrm{~kJ} / \mathrm{mol}$ obtained by Liu and Franke ${ }^{185}$ using a high-level CCSD(T) method; however, at variance to their CCSD(T) calculations, our DFT calculations can be performed in a matter of minutes even on a personal computer.

For cisplatin, we also write out the molecular orbitals after the geometry has been optimized. The molecular orbitals provided by from the non-empirical PBE0/def2TZVP calculations can now be compared to the ones from the semiempirical xtb calculations from section IVA, see figure 6. The frontier orbitals - the highest occupied molecular orbital (HOMO) as well as the lowest unoccupied molecular orbital (LUMO) - from the xtb and NWChem calculations are in good agreement. Also HOMO-3, HOMO-2 and HOMO-1 appear similar; the HOMO-2 and HOMO-1 orbitals are merely switched between the NWChem and xtb calculations.

From the point of view of crystal field theory, the $\mathrm{Pt}(\mathrm{II})$ atom in cisplatin has a square planar coordination and eight $5 d$ electrons. The four HOMOs and the LUMO all involve Pt $5 d$ orbitals. In line with crystal field theory, both NWChem and xtb show that the LUMO involves the $\mathrm{Pt} 5 d_{x^{2}-y^{2}}$ orbital. HOMO-3 in- 


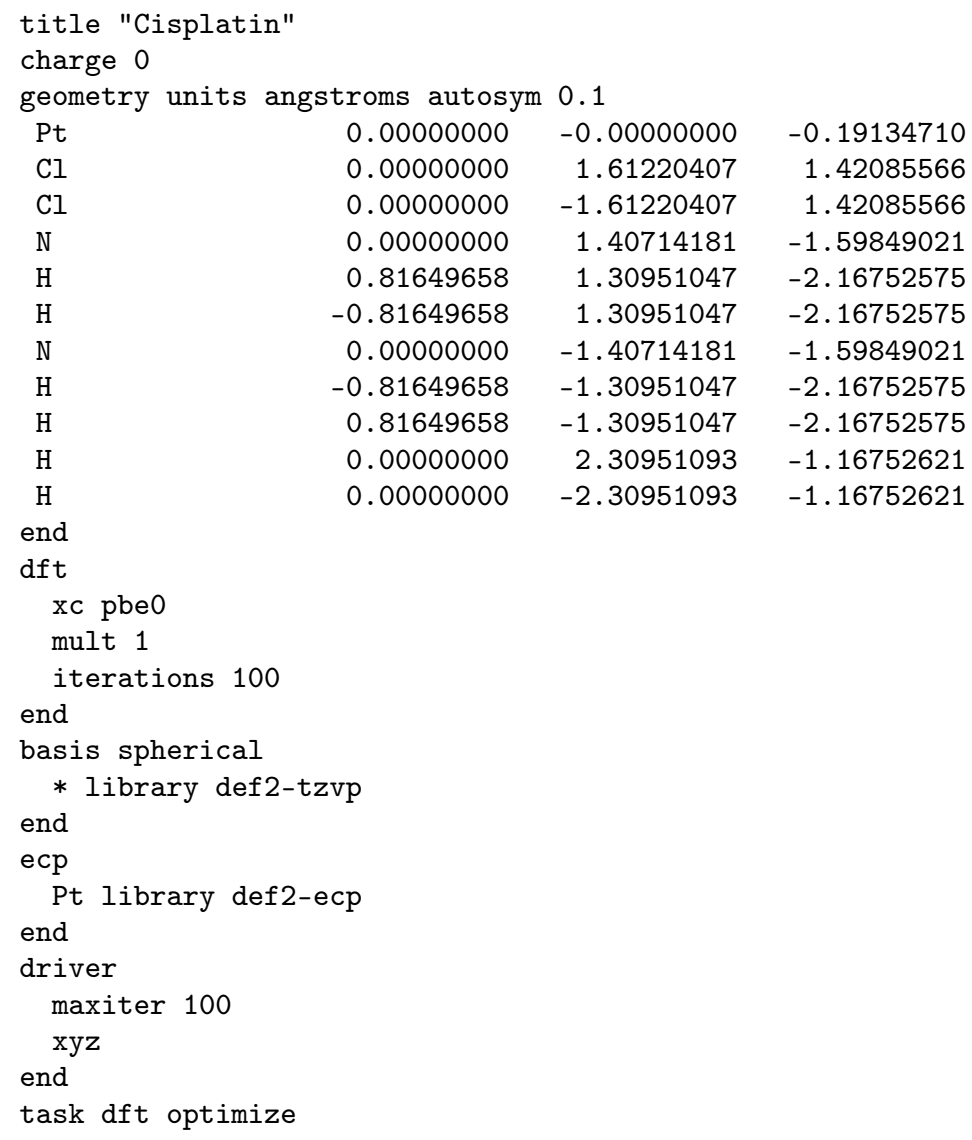

Figure 5. NWChem example: PBE0/def2-TZVP geometry optimization of cisplatin; for transplatin, the nuclear coordinates given in figure 4 are used, instead.

volves the $\mathrm{Pt} 5 d_{z^{2}}$ orbital, while the $5 d_{x y}, 5 d_{x z}$, and $5 d_{y z}$ orbitals contribute to $\mathrm{HOMO}-2, \mathrm{HOMO}-1$, and HOMO. As is clearly seen from the data presented above, the non-empirical PBE0/def2-TZVP and the semiempirical GFN2-xTB level of theory provide a similar description of the frontier orbitals of the Pt(II) complex. Again, the full inputs for the calculations are given in the SI.

\section{Psi4}

While NWChem represented older and more established quantum chemistry codes, Psi4 represents the newer generation of quantum chemistry codes. The origins of PSI4 trace to the PSI3 research code written in $\mathrm{C}++$ for high-accuracy studies on small molecules ${ }^{[7]}$ At variance to PSI3, PSI4 is meant to be a user-friendly, general-purpose code for fast, automated computations on molecules with hundreds of atoms ${ }^{72}$ Psi4 contains a number of computational methods ranging from $\mathrm{HF}$ and DFT to post-HF methods such as Møller-Plesset perturbation theory $\underset{203}{203}$ coupled-cluster theory, ${ }^{204}$ configuration interaction theory, orbital-optimized correlation methods, symmetry-adapted perturbation theory, multireference methods etc..$^{72}$ Although the core of the program is still in $\mathrm{C}++$, PSI4 has thorough Python interfaces and can be used either as a traditional quantum chemistry program with input files, or directly from Python.

We will demonstrate the use of Psi4 in the context of two common exercises in elementary courses on computational chemistry: a conformational study of methylcyclohexane and the reproduction of the molecular geometry of the chromyl fluoride $\left(\mathrm{CrO}_{2} \mathrm{~F}_{2}\right)$ molecule, with a special consideration to the one-electron basis set. We again focus on the def2 family of basis sets that was introduced in section IVB.

\section{Methylcyclohexane}

Starting out with the conformational study of methylcyclohexane, the workflow is as follows. First, the molecule is built in a molecular editor such as Avogadro, IQmol or Jmol, and the drawn molecular structure is preoptimized using a force field available in the editor; the goal of the preoptimization is merely to ensure that the bond lengths are realistic so that the electronic structure calculations during the geometry optimization converge without problems, and so that the bonding pattern does not change. 


\section{NWChem}
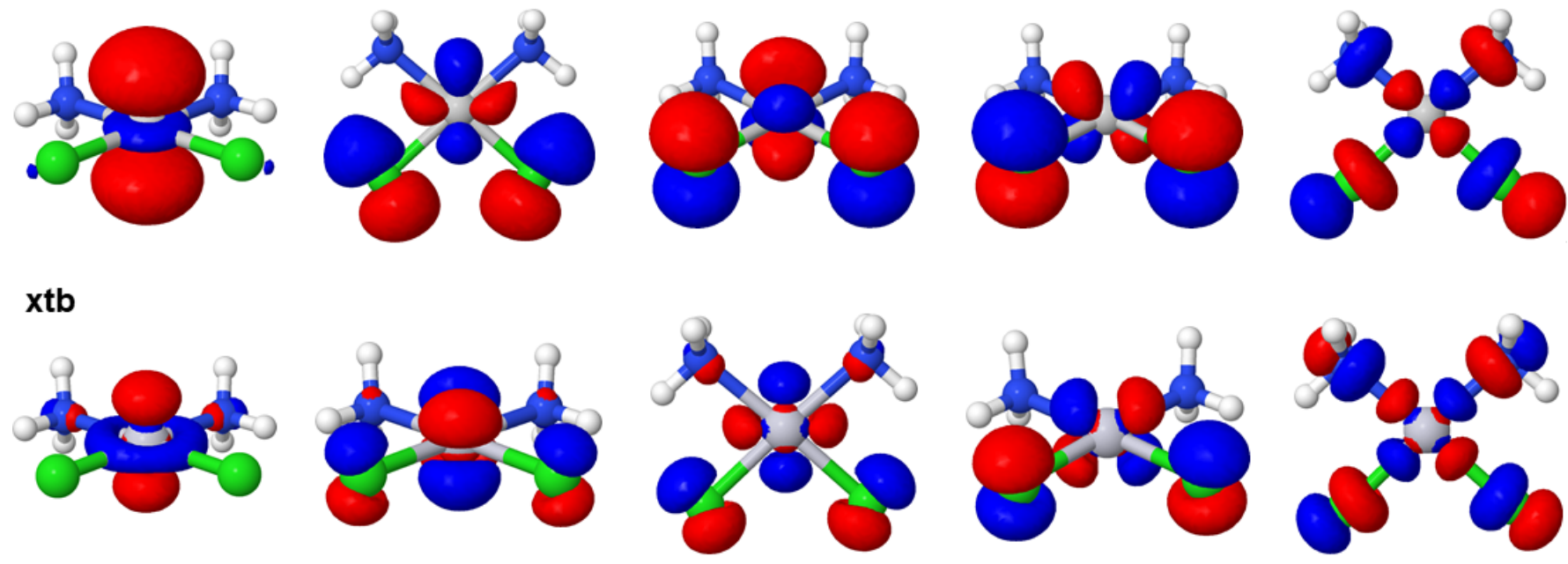

HOMO - 3

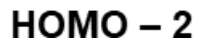

HOMO - 1

HOMO

LUMO

Figure 6. The four highest occupied MOs (HOMOs) and the lowest unoccupied MO (LUMO) of cisplatin as obtained from NWChem (PBE0/def2-TZVP) and xtb (GFN2-xTB). The color code for the nuclei is the same as in figure2, while red and blue denote positive and negative orbital amplitudes, respectively (note that the overall sign of the orbital can be freely chosen). The isovalue used for the orbitals is 0.04 electrons $/$ Bohr $^{3}$.

In the next step, the molecular structure is reoptimized with xtb, and a conformational search is carried out with xtb with the CREST program [Conformer-Rotamer Ensemble Sampling Tool] which has been shown to reproduce conformational ensembles to good accuracy! 205$][207$ Again, the SI includes short tutorials for installing and using the CREST code, which employs xtb to carry out conformational searches of molecules.206

The six conformers found by CREST are then reoptimized in Psi4 using the PBE0/def2-TZVP 79|188|189|196 level of theory introduced above in section IVB Psi4 employs density fitting $208 \sqrt[212]{2 y}$ by default; this means that the universal fitting basis for Hartree-Fock calculations ${ }^{213}$ is used in the calculation. The Psi4 input file for the first conformer is shown in figure 7. The inputs for the other molecules are analogous and shall not be repeated here; they are, however, available in the SI.

With the PBE0/def2-TZVP optimized geometries at hand for each of the six conformers which are given out by CREST in increasing order in energy, we perform single-point calculations on each conformer in a variety of basis sets; the resulting energy differences to the lowestenergy conformer $(\# 1)$ are given in table I In addition to the def2 family, we also have included data for the MINAO basis consisting of the minimal-basis HartreeFock orbitals extracted from the triple- $\zeta$ cc-pVTZ basis set, 214 as well as the STO-3G and STO-6G basis sets which are 3-Gaussian and 6-Gaussian function expansions of a minimal-basis Slater-type orbital (STO) basis set, respectively 192 (It is important to note in this context that not all STO basis sets are minimal: STO basis sets of various sizes ranging up to polarized quadruple$\zeta$ have been reported ${ }^{215 \mid 216}$ and remain widely used for practical calculations in programs employing STO basis sets.)

The data in table $\mathrm{I}$ leads us to the following insights. First, even the minimal basis sets successfully predict the energy ordering of the conformers: although MINAO flips the order of conformers 5 and 6 , it still predicts conformer 1 to be the lowest in energy. Note that this comparison is restricted to the use of fixed geometries; relaxing the geometries in each basis might change the conclusion somewhat. The good performance of the minimal basis sets for this application shows that conformational energies enjoy an excellent degree of error cancellation, which is one of the main motivations for using atomic basis sets in the first place.159

The shortcomings of minimal basis sets are showcased by the large differences between the results obtained with the MINAO and STO- $n$ G basis sets. Minimal basis sets are as small as possible and thereby have very little flexibility: good accuracy for one type of system does not translate to good accuracy in another system, and minimal basis sets generally have poor predictive power for chemistry $121 \mid 122$ MINAO is derived from atomic calculations only, and is thereby fully biased towards atoms, while the Slater-type orbital basis used by Hehre, Stewart, and Pople ${ }^{192}$ is optimized for an average molecular environment, which is reflected in the slightly improved results in table [. The bias towards molecules means that the STO- $n \mathrm{G}$ basis sets are not as good for isolated atoms.

It is generally preferable to use larger and more flexible basis sets in applications, which guarantee a uniform accuracy for all types of systems, and to try to converge results to the complete basis set limit. This means controllably removing the error made in the one-electron ba- 


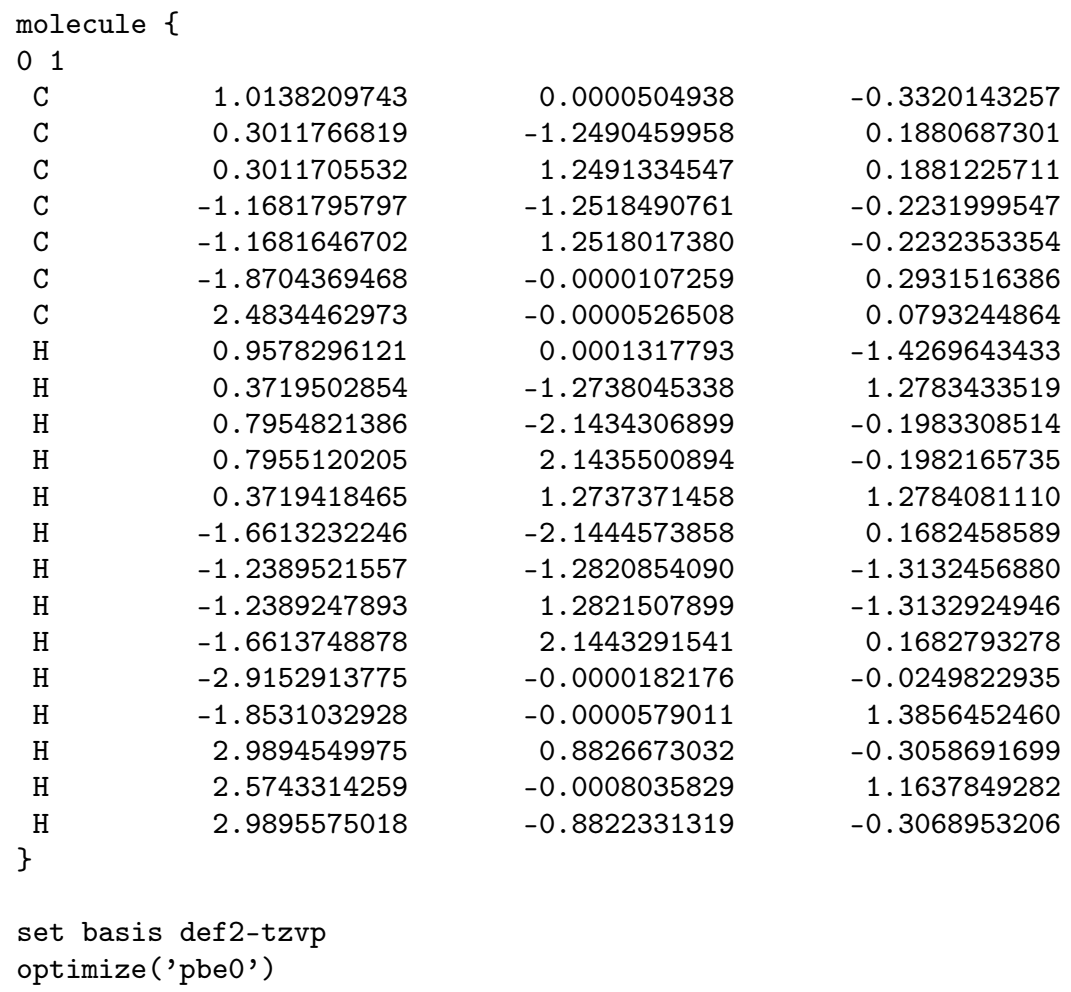

Figure 7. Psi4 example: PBE0/def2-TZVP geometry optimization for the lowest-lying methylcyclohexane conformer.

sis set approximation until the error becomes negligible either in absolute value, or in comparison to the other sources of error in the calculation, such as the error inherent in the employed density functional approximation, for example.

As has already been previously discussed, the smallest reasonable basis for general applications is def2-SV $(\mathrm{P})$. It predicts conformational energies roughly within 0.3 $\mathrm{kcal} / \mathrm{mol}$ compared to the converged quadruple- $\zeta$ values, as can be seen from table I] As shown by the comparison between the def2-SV(P) and def2-SVP data, the role of polarization functions on hydrogen is small for the studied conformational energies.

Systematically more converged energies are obtained by going to the triple- $\zeta$ def2-TZVP basis and the quadruple- $\zeta$ def2-QZVP basis. The data show that already the triple- $\zeta$ calculations are converged to 0.01 $\mathrm{kcal} / \mathrm{mol}$ in the relative energies, demonstrating the usefulness of modern, systematic basis set families: the complete basis set limit can be reached simply by using larger and larger basis sets.

For comparison, table II also includes data for the GFN2-xTB method ${ }^{182} \mathrm{~A}$ visual assessment of the data confirms that GFN2-xTB correctly reproduces the energy ordering of the conformers even with the used PBE0/def2-TZVP geometries, and that the relative energies are reproduced at an accuracy comparable to the minimal basis set calculations, with the converged PBE0/def2-QZVP data as reference. This data emphati- cally suggests that historical applications of minimal basis sets in quantum chemistry can be straightforwardly replaced with modern semiempirical calculations with $\mathrm{xtb}$, for instance, which have much lower computational cost.

Studying a single molecular geometry is in general insufficient, if the molecule has the potential for multiple low-lying conformers. The data in table 1 demonstrates the importance of proper conformational sampling in applications to thermochemistry or chemical reactions, for instance: in the case of methylcyclohexane, insufficient conformational sampling can cause errors of up to 7 $\mathrm{kcal} / \mathrm{mol}$ which may easily surpass the error arising from the level of theory or the basis set.

\section{Geometry of chromyl fluoride}

For a somewhat more complicated example, we study the equilibrium geometry of chromyl fluoride $\left(\mathrm{CrO}_{2} \mathrm{~F}_{2}\right)$ at various levels of DFT, which is known to be surprisingly accurate for simple transition metal complexes. 217 $\mathrm{CrO}_{2} \mathrm{~F}_{2}$ assumes a tetrahedral geometry. Again, the workflow is to build the molecule in a molecular editor, preoptimize the molecular geometry with xtb, and then run the geometry optimizations in Psi4; however, now the optimization is done separately for each basis set in contrast to the procedure used in section IV C 1 .

For this study, we choose the GFN1-xTB ${ }^{218}$ and 


\begin{tabular}{lllcccc} 
method & \multicolumn{7}{c}{$N_{\text {bf }}$ conformer 2} & conformer 3 & conformer 4 & conformer 5 & conformer 6 \\
\hline \hline PBE0/STO-3G & 49 & 1.19 & 2.99 & 1.18 & 5.54 & 5.78 \\
PBE0/STO-6G & 49 & 1.25 & 2.97 & 1.24 & 5.57 & 5.84 \\
PBE0/MINAO & 49 & 0.86 & 2.46 & 0.84 & 5.11 & 5.05 \\
PBE0/def2-SV(P) & 126 & 2.00 & 3.36 & 2.00 & 6.62 & 7.07 \\
PBE0/def2-SVP & 168 & 1.97 & 3.32 & 1.97 & 6.57 & 7.01 \\
PBE0/def2-TZVP & 301 & 2.10 & 3.04 & 2.10 & 6.31 & 6.74 \\
PBE0/def2-QZVP & 819 & 2.11 & 3.03 & 2.11 & 6.31 & 6.73 \\
\hline GFN2-xTB & & 1.35 & 2.58 & 1.35 & 5.21 & 5.19
\end{tabular}

Table I. Relative energies $\Delta E^{\text {conformer } n}=E^{\text {conformer } n}-E^{\text {conformer } 1}$ in $\mathrm{kcal} / \mathrm{mol}$ and number of basis functions $N_{\mathrm{bf}}$ for the methylcyclohexane conformers according to PBE0 calculations with various basis sets, evaluated at the PBE0/def2-TZVP optimized geometries. For comparison, GFN2-xTB data is also included.

GFN2-xTB ${ }^{182}$ semiempirical methods as well as a set of nonempirical density functionals: the PerdewWang 1992 (PW92) local density approximation (LDA) 1361219220 the Perdew-Burke-Ernzerhof (PBE) GGA,,$\sqrt[79]{ }$ as well as the $\mathrm{r}^{2} \mathrm{SCAN}$ meta-GGA functional that represents the state of the art in non-empirical density functionals. $\frac{221222}{2}$ The geometry optimizations are undertaken with very tight convergence thresholds to ensure benchmark quality geometries.

Density fitting is again used in these calculations. As we only consider density functionals that do not contain exact exchange in this application, smaller auxiliary basis sets optimized for reproducing only Coulomb interactions could be employed, 223 however, for simplicity we stick to using the Psi4 default which is to use the larger auxiliary basis sets $\$ 213$ that also work in the presence of exact exchange.

The results shown in table 1 demonstrate that while the STO- $n \mathrm{G}$ minimal basis sets $192 \mid 224$ yield relatively poor geometries compared to the experimental values from refs. 225] and 226, already the split-valence def2$\mathrm{SV}(\mathrm{P})$ basis set ${ }^{196}$ leads to bond lengths that are converged to $0.03 \AA$ and fractions of a degree in angles. The differences become smaller, that is, the bond lengths and angles become more converged going to the larger basis sets, with the differences between the def2-TZVP and def2-QZVP results being already negligible.

The bond lengths from the PBE/def2-QZVP calculations are in excellent agreement with the older experimental values from ref. 225, the bond angles are in reasonable agreement with the experimental data from the same reference. $\mathrm{r}^{2} \mathrm{SCAN} /$ def2-QZVP, in turn, is in excellent agreement with the newer experimental bond lengths from ref. 226.

\section{Quantum Espresso}

Quantum Espresso (QE) is an integrated suite of FOSS codes for electronic structure calculations based on DFT, plane waves, and pseudopotentials. The QE distribution consists of a set of core components and programs, set of plug-ins for more advanced tasks, and a number of third-party packages designed to be interoperable with the core components. QE can be used to study the geometries, energetics, thermodynamics, electronic properties, response properties, spectroscopic properties, and transport properties of solid-state materials. The Supporting Information includes step-by-step guidelines for installing QE and using it to study two polymorphs of zinc(II) sulfide, $\mathrm{ZnS}$.

ZnS crystallizes in two principal forms, sphalerite and wurtzite (figure 8). Sphalerite is a naturally occurring mineral belonging to the cubic crystal system with space group $F[-] 43 m$ (No. 216). Both $\mathrm{Zn}$ and S atoms are tetrahedrally coordinated in the sphalerite structure and the crystal structure can be considered as a diamond lattice with two atom types. Wurtzite is also a naturally occurring mineral and it can be considered as a hexagonal polymorph of sphalerite, crystallizing in the space group $P 6_{3} m c$ (No. 186). The coordination with nearest and next-nearest neighbors in wurtzite is identical to that in sphalerite. The first structural differences between the two polymorphs arise only in the third shell of neighbors ${ }^{[227}$ From a thermodynamical point of view, sphalerite is the low-temperature $\mathrm{ZnS}$ polymorph in bulk form and the transition temperature to wurtzite is $1293 \pm 10 \mathrm{~K}^{[228}$ Wurtzite- $\mathrm{ZnS}$ is thus metastable at room temperature, but it is found in nature and can also be produced synthetically.

The illustrative QE calculations are carried out with the non-empirical PBE exchange-correlation functional. ${ }^{79}$ To run the calculations with QE, we need pseudopotentials that have been developed for this functional. Here we use the ultrasoft Garrity-BennettRabe-Vanderbilt (GBRV) pseudopotentials, which form a highly accurate and computationally inexpensive open-source pseudopotential library that has been designed and optimized for use in high-throughput DFT calculations. ${ }^{229}$ The main attractive feature of the GBRV pseudopotentials is that they have been designed for relatively small plane wave cutoffs of 40 Rydberg for wave functions, and 200 Rydberg for the charge density and potential, 229 which results in relatively low 


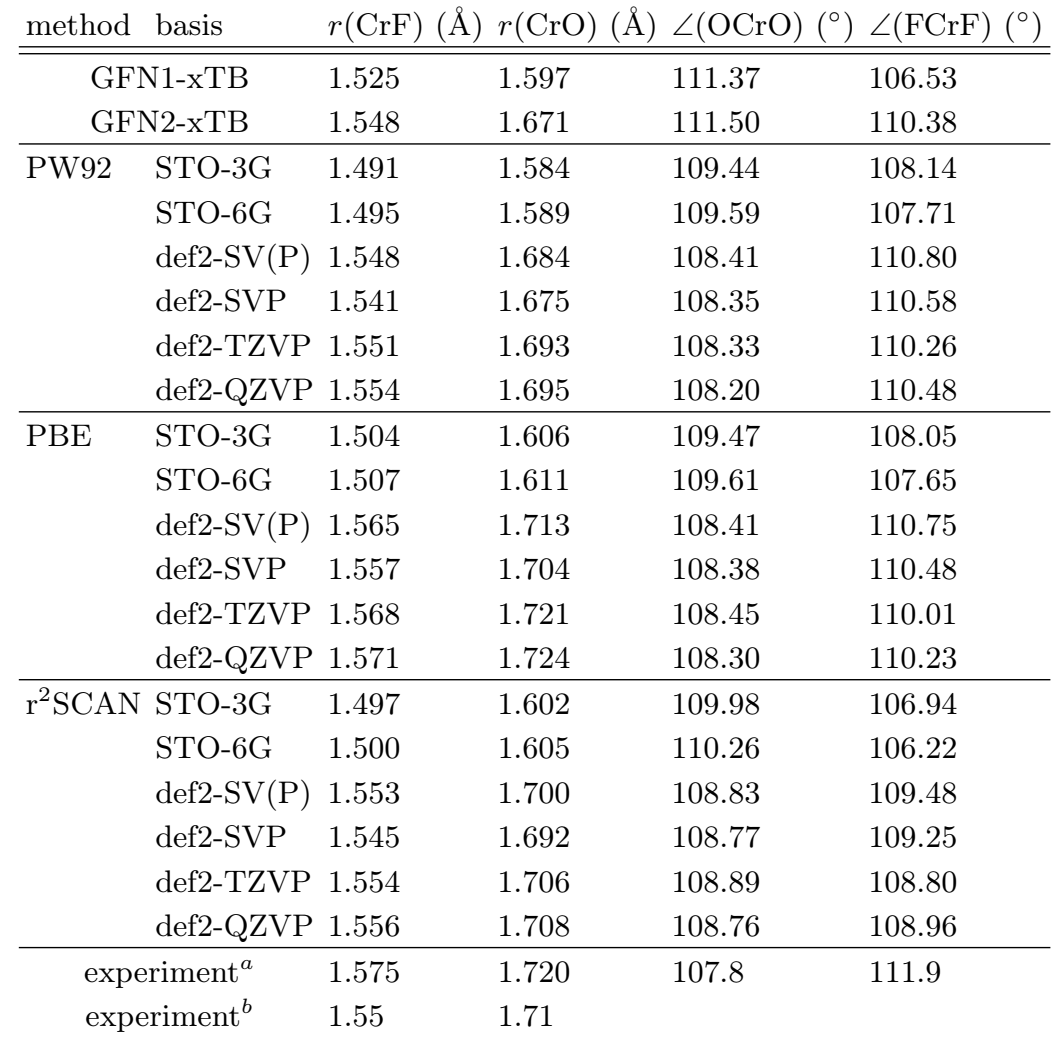

Table II. Geometric parameters of chromyl fluoride $\left(\mathrm{CrO}_{2} \mathrm{~F}_{2}\right)$ at various levels of theory. ${ }^{a}$ Experimental values from ref. 225 . ${ }^{b}$ Experimental values from ref. 226.
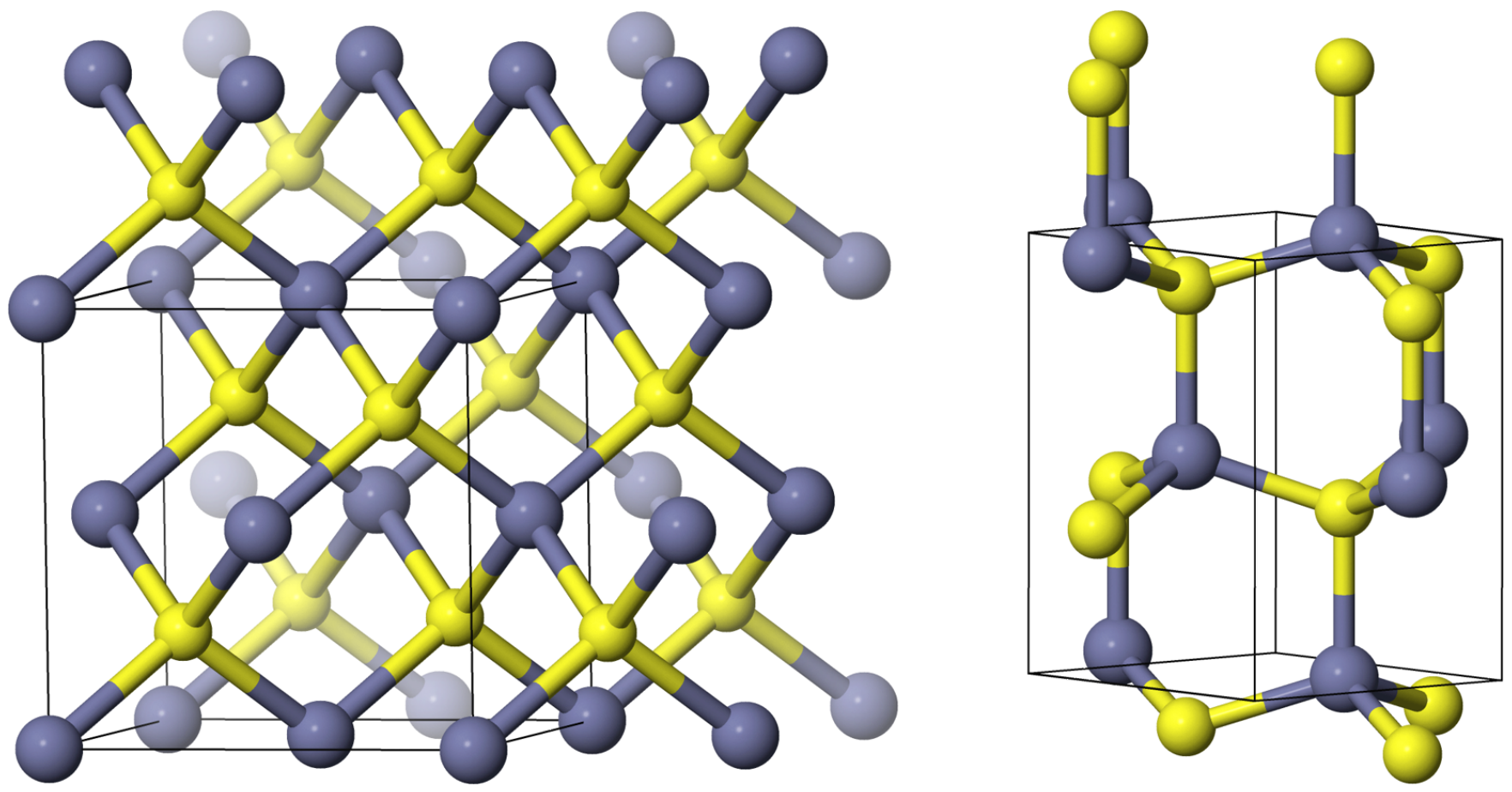

Figure 8. Two polymorphs of ZnS: sphalerite (left) and wurtzite (right). Zinc atoms in blue, sulfur atoms in yellow. For wurtzite, the $c$-axis points upwards. 


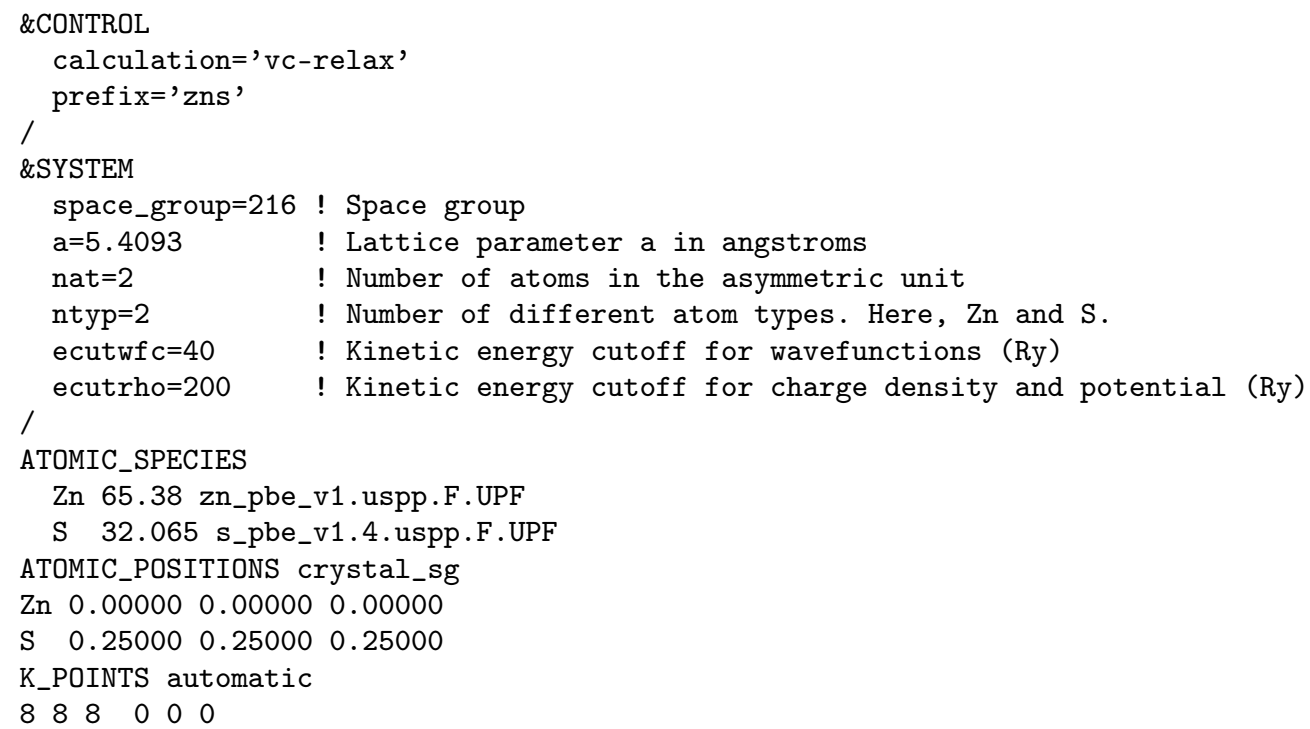

Figure 9. Quantum Espresso example: Geometry optimization of sphalerite-ZnS with PBE functional and GBRV pseudopotentials. Fully annotated input files can be found from the SI.

computational costs.

To study sphalerite-ZnS and wurtzite-ZnS with QE, we need their crystal structures. A good source for crystal structure data is the Crystallography Open Database $(\mathrm{COD}) \stackrel{230}{2}$ which is where we obtained the structures in the Crystallographic Information File (CIF) format; the COD structures are available in the Supporting Information.

There are several ways in which the crystal structures can be entered in QE input files. In the example here, we have directly used the crystallographic information to create an input file, which is shown in figure 9, a very helpful resource for building QE input files is afforded by the QE input generator and structure visualizer provided by the Materials Cloud.231

\section{Optimal geometry}

Before attempting any calculations, it is important to determine how dense a sampling of the reciprocal space (k-sampling) is needed to describe the materials sufficiently accurately. The convergence tests described in the Supporting Information show that a $8 \times 8 \times 8$ MonkhorstPack ${ }^{232} \mathbf{k}$-point mesh leads to a truncation error smaller than $1 \mathrm{meV}$ for sphalerite-ZnS. A comparable k-point spacing is then also used for wurtzite-ZnS.

The geometry optimization of sphalerite-ZnS finishes in a few minutes, while the wurtzite-ZnS may take tens of minutes when run on a single processor core. The optimized lattice parameters are in good agreement with the experimental lattice parameters found on COD. The optimized lattice parameters are $a=5.447 \AA$ for sphaleriteZnS and $a=3.846 \AA$ and $c=6.304 \AA$ for wurtzite$\mathrm{ZnS}$, whereas the experimental lattice parameters are $a=5.4093 \AA$ for sphalerite-ZnS and $a=3.811 \AA$ and $c=6.234 \AA$ for wurtzite-ZnS ${ }^{230}$ This means that the computations overestimate the lattice parameters by approximately $1 \%$ over the experiment.

The energy comparison of the optimized sphalerite$\mathrm{ZnS}$ and wurtzite-ZnS structures shows that the total energies differ by only $0.6 \mathrm{~kJ} / \mathrm{mol}$ per formula unit. This value is in good agreement with Cardona et al. 233 who reported an energy difference of less than $0.008 \mathrm{eV}(0.8$ $\mathrm{kJ} / \mathrm{mol}$ ) per formula unit from LDA and GGA calculations on $\mathrm{ZnS}$ polymorphs. The energy difference is so small, because the crystal structures are so similar: differences arise only in the third-nearest neighbor shell. Note that so far we have only compared electronic total energies; Gibbs free energies should be considered instead for a full understanding of the thermodynamics, but this is beyond the scope of this work.

\section{Band structure}

The second practical example illustrates how the electronic band structure of sphalerite- $\mathrm{ZnS}$ can be calculated and plotted with QE. In any band structure calculation, the band path in the reciprocal space has to be defined in terms of k-points. The band path depends on the Bravais lattice of the crystal structure. An excellent source for band paths is the SeeK-path service, $\stackrel{234}{2}$ which readily provides crystal-structure-based band paths for several program packages. Here, we use the face centered cubic (FCC) band path from Setyawan and Curtarolo ${ }^{235}$, and the resulting electronic band structure of sphalerite-ZnS is illustrated in figure 10 .

From the band structure plot in figure 10 , we can see that sphalerite-ZnS has a direct band gap of about $2 \mathrm{eV}$ 


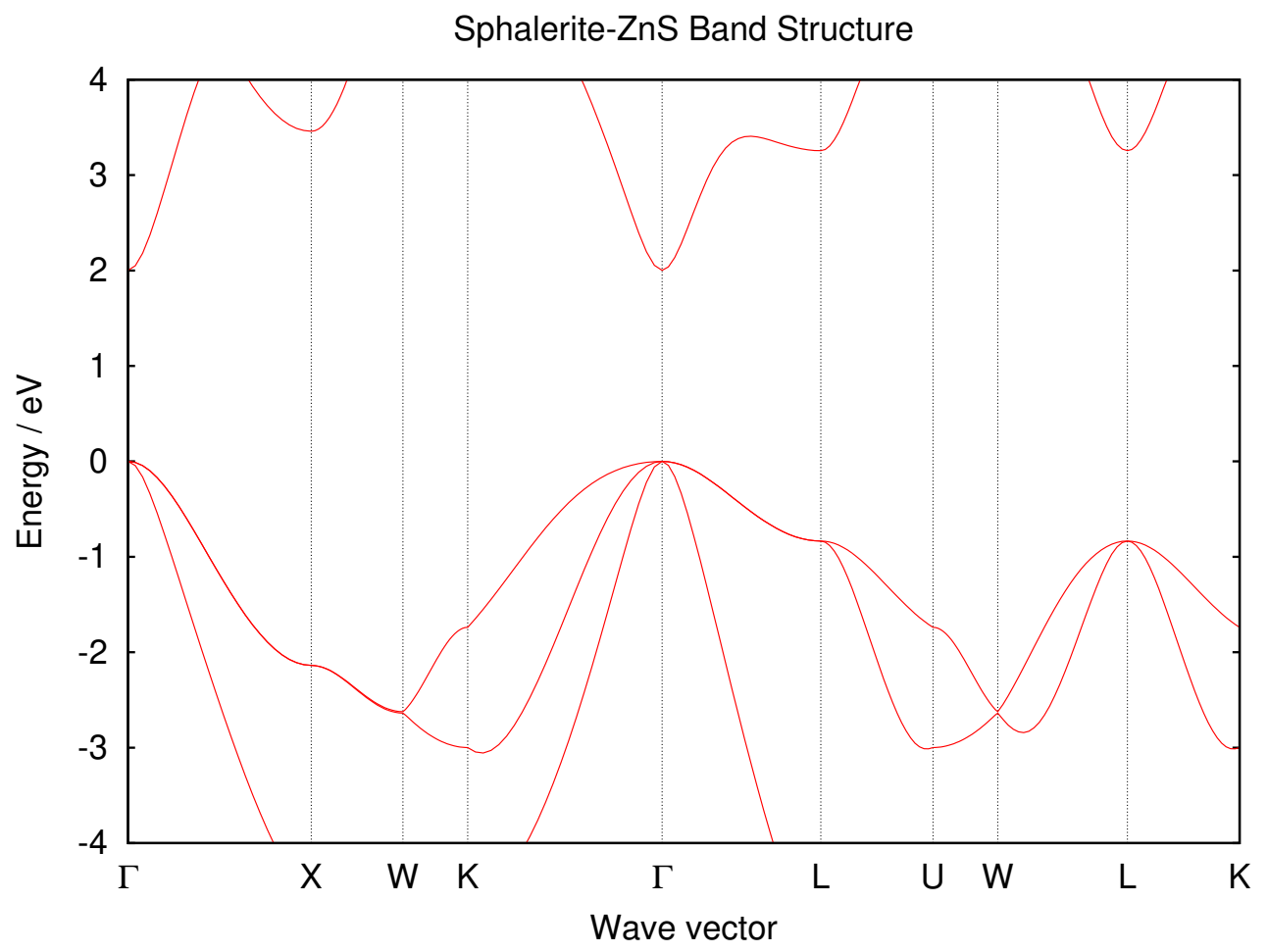

Figure 10. Electronic band structure of sphalerite-ZnS obtained with PBE functional and GBRV pseudopotentials.

at the $\Gamma$ point when using the PBE functional and the GBRV pseudopotentials. The band structure in figure 10 is in good agreement with the PBE band structure available in the Materials Project ${ }^{236}$ However, the PBE calculations severely underestimate the experimental band gap measured at $10 \mathrm{~K}$, which is about $3.8 \mathrm{eV} \stackrel{237}{\text { The }}$ agreement with experiment could be improved for example with the DFT $+\mathrm{U}$ approach or with hybrid density functionals which are outside the scope of this work.

\section{SUMMARY AND CONCLUSIONS}

We have argued that free and open source software (FOSS) allows for a bring your own device (BYOD) approach to the teaching of computational chemistry, and finally affords computational chemistry for the masses, thereby also democratizing the science of computational chemistry. The distributed BYOD approach to computational chemistry also supports the delivery of massive open online courses (MOOCs), avoiding the need to organize computing resources for a large number of students in a cost-effective and secure way. We have briefly reviewed the current selection of FOSS programs for electronic structure calculations, and illustrated the installation and practical use of several programs for computational chemistry education on personal computers. As the technical barriers for running quantum chemical calculations on personal laptops have practically van- ished, educators can focus on content creation and developing practices for sharing and co-creating computational chemistry teaching material as Open Educational Resources ${ }^{238}$ The Psi4Education project ${ }^{5}$ is one such attempt at open teaching materials; we hope such materials become more readily available and more thoroughly used in the future.

\section{SUPPORTING INFORMATION}

Full input and output files for the practical examples discussed in this work, together with step-by-step instructions for installing and running the required program packages on Linux, macOS, or Windows Subsystem for Linux. The supporting information is also available as a git repository $\frac{181}{181}$

\section{ACKNOWLEDGMENTS}

We thank Paul Saxe and Jonathan Moussa for invaluable comments on the manuscript. A. J. K. thanks Business Finland for Co-Innovation funding (Grant No. $3767 / 31 / 2019)$. 


\section{REFERENCES}

${ }^{1} \mathrm{P}$. Westmoreland, Applying Molecular and Materials Modeling 1st ed. (Springer Netherlands, 2002).

${ }^{2}$ M. Head-Gordon and E. Artacho, "Chemistry on the computer," Phys. Today 61, 58-63 (2008)

${ }^{3}$ P. Deglmann, A. Schäfer, and C. Lennartz, "Application of quantum calculations in the chemical industry - An overview," Int. J. Quantum Chem. 115, 107-136 (2015)

${ }^{4}$ H. Weiß, P. Deglmann, P. J. in 't Veld, M. Cetinkaya, and E. Schreiner, "Multiscale materials modeling in an industrial environment," Annu. Rev. Chem. Biomol. Eng. 7, 65-86 (2016) ${ }^{5}$ R. C. Fortenberry, A. R. McDonald, T. D. Shepherd, M. Kennedy, and C. D. Sherrill, "PSI4Education: Computational chemistry labs using free software," in The Promise of Chemical Education: Addressing our Students' Needs (American Chemical Society, 2015) pp. 85-98.

${ }^{6}$ A. Grushow and M. S. e. Reeves, Using Computational Methods To Teach Chemical Principles (American Chemical Society, 2019).

${ }^{7}$ B. J. Esselman and N. J. Hill, "Integration of computational chemistry into the undergraduate organic chemistry laboratory curriculum," J. Chem. Educ. 93, 932-936 (2016)

${ }^{8}$ L. L. Winfield, K. McCormack, and T. Shaw, "Using iSpartan to support a student-centered activity on alkane conformations," J. Chem. Educ. 96, 89-92 (2018)

${ }^{9}$ B. J. Esselman and N. J. Hill, "Integrating computational chemistry into an organic chemistry laboratory curriculum using webmo," in Using Computational Methods To Teach Chemical Principles, Chap. 11, pp. 139-162.

${ }^{10} \mathrm{~J}$. A. Phillips, "Modeling reaction energies and exploring noble gas chemistry in the physical chemistry laboratory," in Using Computational Methods To Teach Chemical Principles Chap. 4, pp. 33-50.

${ }^{11}$ M. S. Reeves, H. L. Berghout, M. J. Perri, S. M. Singleton, and R. M. Whitnell, "How can you measure a reaction enthalpy without going into the lab?" in Using Computational Methods To Teach Chemical Principles, Chap. 5, pp. 51-63.

${ }^{12}$ S. R. Martini and C. J. Hartzell, "Integrating computational chemistry into a course in classical thermodynamics," Journal of Chemical Education 92, 1201-1203 (2015).

${ }^{13}$ K. M. Stocker, "Using electronic structure calculations to investigate the kinetics of gas-phase ammonia synthesis," in Using Computational Methods To Teach Chemical Principles Chap. 3, pp. 21-32.

${ }^{14}$ H. D. Snyder and T. G. Kucukkal, "Computational chemistry activities with avogadro and ORCA," J. Chem. Educ. 98, 1335$1341(2021)$

${ }^{15}$ G. C. Hoover, A. P. Dicks, and D. S. Seferos, "Upper-year materials chemistry computational modeling module for organic display technologies," J. Chem. Educ. 98, 805-811 (2021)

${ }^{16} \mathrm{P}$. Y. Furlan and E. T. Bell-Loncella, "Integrating computation and visualization to enhance learning IR spectroscopy in the general chemistry laboratory: Computer-assisted learning of IR spectroscopy," Spectrosc. Lett. 43, 618-625 (2010).

${ }^{17} \mathrm{~W}$. R. Martin and D. W. Ball, "Using computational chemistry to extend the acetylene rovibrational spectrum to $\mathrm{c}_{2} \mathrm{t}_{2}$," in Using Computational Methods To Teach Chemical Principles Chap. 8, pp. 93-107.

${ }^{18}$ T. C. DeVore, "Introducing quantum calculations into the physical chemistry laboratory," in Using Computational Methods To Teach Chemical Principles, Chap. 9, pp. 109-125.

${ }^{19} \mathrm{JCE}$ staff, "Computational chemistry for the masses," J. Chem. Educ. 73, 104 (1996)

${ }^{20}$ A. Grushow and M. S. Reeves, "Using computational methods to teach chemical principles: Overview," in Using Computational Methods To Teach Chemical Principles (2019) Chap. 1, pp. 110 .

${ }^{21}$ WebMO, "A web-based interface to computational chemistry packages," https://www.webmo.net/, accessed 8 May 2021.
${ }^{22}$ W. F. Polik and J. R. Schmidt, "WebMO : Web-based computational chemistry calculations in education and research," [ (2021), 10.1002/wcms.1554

${ }^{23}$ M. J. Perri, M. Akinmurele, and M. Haynie, "Chem compute science gateway: An online computational chemistry tool," in Using Computational Methods To Teach Chemical Principles Chap. 7, pp. 79-92.

${ }^{24}$ R. Kobayashi, T. P. M. Goumans, N. O. Carstensen, T. M. Soini, N. Marzari, I. Timrov, S. Poncé, E. B. Linscott, C. J. Sewell, G. Pizzi, F. Ramirez, M. Bercx, S. P. Huber, C. S. Adorf, and L. Talirz, "Virtual computational chemistry teaching laboratories-hands-on at a distance," J. Chem. Educ. 98, 3163-3171 (2021)

${ }^{25} \mathrm{~S}$. Schwalbe, L. Fiedler, J. Kraus, J. Kortus, K. Trepte, and S. Lehtola, "PyFLOSIC: Python-based Fermi-Löwdin orbital self-interaction correction," J. Chem. Phys. 153, 084104 (2020)

${ }^{26}$ A. I. Krylov, J. M. Herbert, F. Furche, M. Head-Gordon, P. J. Knowles, R. Lindh, F. R. Manby, P. Pulay, C.-K. Skylaris, and H.-J. Werner, "What is the price of open-source software?" J. Phys. Chem. Lett. 6, 2751-2754 (2015).

${ }^{2 /}$ C. R. Jacob, "How open is commercial scientific software?" J. Phys. Chem. Lett. 7, 351-353 (2016)

${ }^{28} \mathrm{~L}$. Li, "Why should anyone become a scientist? the ideal of science and its importance," J. Chem. Educ. 76, 20 (1999).

${ }^{29}$ P. Azoulay, C. Fons-Rosen, and J. S. G. Zivin, "Does science advance one funeral at a time?" Am. Econ. Rev. 109, 2889$2920(2019)$

${ }^{30}$ J. Giles, "Software company bans competitive users," Nature 429, 231-231 (2004)

${ }^{11}$ A. G. Smart, "The war over supercooled water," Physics Today (2018).

32 J. C. Palmer, A. Haji-Akbari, R. S. Singh, F. Martelli, R. Car, A. Z. Panagiotopoulos, and P. G. Debenedetti, "Comment on "The putative liquid-liquid transition is a liquid-solid transition in atomistic models of water" [I and II: J. Chem. Phys. 135, 134503 (2011); J. Chem. Phys. 138, 214504 (2013)]," J. Chem. Phys. 148, 137101 (2018)

${ }^{35}$ Open Source Initiative, "The open source definition," https://opensource.org/osd, accessed May 132021.

${ }^{34}$ Free Software Foundation, "What is free software?" https://www.gnu.org/philosophy/free-sw.html.en, accessed May 132021.

${ }^{35}$ M. T. Stahl, "Open-source software: not quite endsville," Drug Discovery Today 10, 219-222 (2005)

${ }^{36}$ J. D. Gezelter, "Open source and open data should be standard practices," J. Phys. Chem. Lett. 6, 1168-1169 (2015).

${ }^{37} \mathrm{~K}$. Hinsen, "Computational science: shifting the focus from tools to models," F1000Research 3, 101 (2014)

${ }^{38}$ Git community, "Git, a free and open source distributed version control system," https://git-scm.com/, accessed 20 May 2021.

${ }^{39}$ GitHub, Inc., "Github collaboration platform," https://github.com/, accessed 20 May 2021.

${ }^{40}$ GitLab, Inc., "Gitlab collaboration platform," https://gitlab.com/, accessed 20 May 2021.

${ }^{41}$ European Organization For Nuclear Research and OpenAIRE, "Zenodo," (2013).

${ }^{42}$ J. Swarts, "Open-source software in the sciences: The challenge of user support," J. Bus. Tech. Commun. 33, 60-90 (2018).

${ }^{43}$ A. Dalke, "The chemfp project," J. Cheminf. 11, 76 (2019)|

${ }^{44} \mathrm{G}$. Haff, How open source ate software: Understand the open source movement and so much more (2018) pp. 1-180.

${ }^{45} \mathrm{~F}$. Wieber, A. Pisanty, and A. Hocquet, " "we were here before the web and hype...": a brief history of and tribute to the computational chemistry list," J. Cheminf. 10,67 (2018)

${ }^{46}$ D. Constant, L. Sproull, and S. Kiesler, "The kindness of strangers: The usefulness of electronic weak ties for technical advice," Organ. Sci. 7, 119-135 (1996)

${ }^{47}$ K. R. Lakhani and E. von Hippel, "How open source software works: "free" user-to-user assistance," Res. Policy 32, 923-943 (2003) 
${ }^{48}$ A. Schiff, "The economics of open source software: A survey of the early literature," Rev. Netw. Econ. 1, 66-74 (2002).

${ }^{49}$ D. P. Myatt, "Equilibrium selection and public-good provision: The development of open-source software," Oxford Rev. Econ. Pol. 18, 446-461 (2002)

${ }^{50}$ J. P. Johnson, "Open source software: Private provision of a public good," J. Econ. Manage. Strat. 11, 637-662 (2002)

${ }^{51} \mathrm{M}$. Mustonen, "Copyleft- the economics of linux and other open source software," Inf. Econ. Policy 15, 99-121 (2003)

${ }^{52}$ J. Lerner and J. Tirole, "Some simple economics of open source," The Journal of Industrial Economics 50, 197-234 (2003)

${ }^{53} \mathrm{~A}$. Bonaccorsi and C. Rossi, "Why open source software can succeed," Res. Policy 32, 1243-1258 (2003)

${ }^{54}$ R. E. Hawkins, "The economics of open source software for a competitive firm," NETNOMICS: Economic Research and Electronic Networking 6, 103-117 (2004)

J. Bitzer, "Commercial versus open source software: the role of product heterogeneity in competition," Economic Systems 28, 369-381 (2004)

${ }^{56} \mathrm{~J}$. Lerner and J. Tirole, "The economics of technology sharing: Open source and beyond," J. Econ. Perspect. 19, 99-120 (2005)

${ }^{57}$ J. Lerner, "The scope of open source licensing," Journal of Law, Economics, and Organization 21, 20-56 (2005)

${ }^{58}$ J. Bitzer and P. J. H. Schröder, "Bug-fixing and code-writing: The private provision of open source software," Inf. Econ. Policy 17, 389-406 (2005)

${ }^{59}$ J. West and S. Gallagher, "Challenges of open innovation: the paradox of firm investment in open-source software," R and D Management 36, 319-331 (2006)

${ }^{\text {ou} M . ~ A . ~ R o s s i, ~ " D e c o d i n g ~ t h e ~ f r e e / o p e n ~ s o u r c e ~ s o f t w a r e ~ p u z z l e, " ~}$ in The Economics of Open Source Software Development (Elsevier, 2006) pp. 15-55.

${ }^{61} \mathrm{~A}$. Gaudeul, "Do open source developers respond to competition? the LATEX case study," Rev. Netw. Econ. 6, 239-263 (2007)

${ }^{02}$ G. von Krogh and E. von Hippel, "The promise of research on open source software," Manage. Sci. 52, 975-983 (2006).

${ }^{63} \mathrm{~A}$. Hars and S. Ou, "Working for free? motivations for participating in open-source projects," Int. J. Electron. Comm. 6, 25-39 (2002)

${ }^{64}$ J. Bitzer, W. Schrettl, and P. J. H. Schröder, "Intrinsic motivation in open source software development," J. Comp. Econ. 35, 160-169 (2007)

${ }^{65}$ J. Lerner, P. A. Pathak, and J. Tirole, "The dynamics of opensource contributors," Am. Econ. Rev. 96, 114-118 (2006)

${ }^{66}$ C. Fershtman and N. Gandal, "Open source software: Motivation and restrictive licensing," International Economics and Economic Policy 4, 209-225 (2007)

${ }^{6} \mathrm{r}$ J. P. Johnson, "Collaboration, peer review and open source software," Inf. Econ. Policy 18, 477-497 (2006).

${ }^{68} \mathrm{~J}$. Bitzer and P. J. H. Schröder, "The impact of entry and competition by open source software on innovation activity," in The Economics of Open Source Software Development (Elsevier, 2006) pp. 219-246.

69 top500.org, "Top500 operating system statistics," https://www.top500.org/statistics/details/osfam/1/, accessed 6 July 2021.

${ }^{70}$ J. F. Moore and M. P. McCann, "Linux and the Chemist," J. Chem. Educ. 80, 219 (2003)

${ }^{1}$ J. Lehtola, M. Hakala, A. Sakko, and K. Hämäläinen, "ERKALE - A flexible program package for X-ray properties of atoms and molecules," J. Comput. Chem. 33, 1572-1585 (2012)

${ }^{72}$ D. G. A. Smith, L. A. Burns, A. C. Simmonett, R. M. Parrish, M. C. Schieber, R. Galvelis, P. Kraus, H. Kruse, R. Di Remigio, A. Alenaizan, A. M. James, S. Lehtola, J. P. Misiewicz, M. Scheurer, R. A. Shaw, J. B. Schriber, Y. Xie, Z. L. Glick, D. A. Sirianni, J. S. O'Brien, J. M. Waldrop, A. Kumar, E. G. Hohenstein, B. P. Pritchard, B. R. Brooks, H. F. Schaefer, A. Y. Sokolov, K. Patkowski, A. E. DePrince, U. Bozkaya, R. A. King, F. A. Evangelista, J. M. Turney, T. D. Crawford, and C. D.
Sherrill, "Psi4 1.4: Open-source software for high-throughput quantum chemistry," J. Chem. Phys. 152, 184108 (2020)

${ }^{73}$ T. D. Crawford, C. D. Sherrill, E. F. Valeev, J. T. Fermann, R. A. King, M. L. Leininger, S. T. Brown, C. L. Janssen, E. T. Seidl, J. P. Kenny, and W. D. Allen, "PSI3: An open-source Ab Initio electronic structure package," J. Comput. Chem. 28, 1610-1616 (2007)

${ }^{14}$ Q. Sun, X. Zhang, S. Banerjee, P. Bao, M. Barbry, N. S. Blunt, N. A. Bogdanov, G. H. Booth, J. Chen, Z.-H. Cui, J. J. Eriksen, Y. Gao, S. Guo, J. Hermann, M. R. Hermes, K. Koh, P. Koval, S. Lehtola, Z. Li, J. Liu, N. Mardirossian, J. D. McClain, M. Motta, B. Mussard, H. Q. Pham, A. Pulkin, W. Purwanto, P. J. Robinson, E. Ronca, E. R. Sayfutyarova, M. Scheurer, H. F. Schurkus, J. E. T. Smith, C. Sun, S.-N. Sun, S. Upadhyay, L. K. Wagner, X. Wang, A. White, J. D. Whitfield, M. J. Williamson, S. Wouters, J. Yang, J. M. Yu, T. Zhu, T. C. Berkelbach, S. Sharma, A. Y. Sokolov, and G. K.-L. Chan, "Recent developments in the PYSCF program package," J. Chem. Phys. 153, 024109 (2020), arXiv:2002.12531

${ }^{75}$ F. Aquilante, J. Autschbach, A. Baiardi, S. Battaglia, V. A. Borin, L. F. Chibotaru, I. Conti, L. De Vico, M. Delcey, I. Fdez. Galván, N. Ferré, L. Freitag, M. Garavelli, X. Gong, S. Knecht, E. D. Larsson, R. Lindh, M. Lundberg, P. A. Malmqvist, A. Nenov, J. Norell, M. Odelius, M. Olivucci, T. B. Pedersen, L. Pedraza-González, Q. M. Phung, K. Pierloot, M. Reiher, I. Schapiro, J. Segarra-Martí, F. Segatta, L. Seijo, S. Sen, D.-C. Sergentu, C. J. Stein, L. Ungur, M. Vacher, A. Valentini, and V. Veryazov, "Modern quantum chemistry with [Open]Molcas," J. Chem. Phys. 152, 214117 (2020)

${ }^{76}$ J. M. H. Olsen, S. Reine, O. Vahtras, E. Kjellgren, P. Reinholdt, K. O. Hjorth Dundas, X. Li, J. Cukras, M. Ringholm, E. D. Hedegård, R. Di Remigio, N. H. List, R. Faber, B. N. Cabral Tenorio, R. Bast, T. B. Pedersen, Z. Rinkevicius, S. P. A. Sauer, K. V. Mikkelsen, J. Kongsted, S. Coriani, K. Ruud, T. Helgaker, H. J. A. Jensen, and P. Norman, "Dalton Project: A Python platform for molecular- and electronic-structure simulations of complex systems," J. Chem. Phys. 152, 214115 (2020)

${ }^{77}$ E. Aprà, E. J. Bylaska, W. A. de Jong, N. Govind, K. Kowalski, T. P. Straatsma, M. Valiev, H. J. J. van Dam, Y. Alexeev, J. Anchell, V. Anisimov, F. W. Aquino, R. Atta-Fynn, J. Autschbach, N. P. Bauman, J. C. Becca, D. E. Bernholdt, K. Bhaskaran-Nair, S. Bogatko, P. Borowski, J. Boschen, J. Brabec, A. Bruner, E. Cauët, Y. Chen, G. N. Chuev, C. J. Cramer, J. Daily, M. J. O. Deegan, T. H. Dunning, M. Dupuis, K. G. Dyall, G. I. Fann, S. A. Fischer, A. Fonari, H. Früchtl, L. Gagliardi, J. Garza, N. Gawande, S. Ghosh, K. Glaesemann, A. W. Götz, J. Hammond, V. Helms, E. D. Hermes, K. Hirao, S. Hirata, M. Jacquelin, L. Jensen, B. G. Johnson, H. Jónsson, R. A. Kendall, M. Klemm, R. Kobayashi, V. Konkov, S. Krishnamoorthy, M. Krishnan, Z. Lin, R. D. Lins, R. J. Littlefield, A. J. Logsdail, K. Lopata, W. Ma, A. V. Marenich, J. Martin del Campo, D. Mejia-Rodriguez, J. E. Moore, J. M. Mullin, T. Nakajima, D. R. Nascimento, J. A. Nichols, P. J. Nichols, J. Nieplocha, A. Otero-de-la Roza, B. Palmer, A. Panyala, T. Pirojsirikul, B. Peng, R. Peverati, J. Pittner, L. Pollack, R. M. Richard, P. Sadayappan, G. C. Schatz, W. A. Shelton, D. W. Silverstein, D. M. A. Smith, T. A. Soares, D. Song, M. Swart, H. L. Taylor, G. S. Thomas, V. Tipparaju, D. G. Truhlar, K. Tsemekhman, T. Van Voorhis, Á. Vázquez-Mayagoitia, P. Verma, O. Villa, A. Vishnu, K. D. Vogiatzis, D. Wang, J. H. Weare, M. J. Williamson, T. L. Windus, K. Woliński, A. T. Wong, Q. Wu, C. Yang, Q. Yu, M. Zacharias, Z. Zhang, Y. Zhao, and R. J. Harrison, "NWChem: Past, present, and future," J. Chem. Phys. 152, $184102(2020)$

${ }^{r}$ S. Lehtola, C. Steigemann, M. J. T. Oliveira, and M. A. L. Marques, "Recent developments in LIBXC - A comprehensive library of functionals for density functional theory," SoftwareX 7, 1-5 (2018)

${ }^{79}$ J. P. Perdew, K. Burke, and M. Ernzerhof, "Generalized Gradient Approximation Made Simple," Phys. Rev. Lett. 77, 3865- 
3868 (1996)

${ }^{8}$ P. J. Stephens, F. J. Devlin, C. F. Chabalowski, and M. J. Frisch, "Ab Initio Calculation of Vibrational Absorption and Circular Dichroism Spectra Using Density Functional Force Fields," J. Phys. Chem. 98, 11623-11627 (1994)

${ }^{81}$ J. Sun, A. Ruzsinszky, and J. Perdew, "Strongly Constrained and Appropriately Normed Semilocal Density Functional," Phys. Rev. Lett. 115, 036402 (2015)

${ }^{82}$ P. Giannozzi, O. Baseggio, P. Bonfà, D. Brunato, R. Car, I. Carnimeo, C. Cavazzoni, S. de Gironcoli, P. Delugas, F. Ferrari Ruffino, A. Ferretti, N. Marzari, I. Timrov, A. Urru, and S. Baroni, "QuAntum ESPRESSO toward the exascale," J. Chem. Phys. 152, 154105 (2020)

${ }^{8}$ S. Lehtola, "Fully numerical Hartree-Fock and density functional calculations. II. Diatomic molecules," Int. J. Quantum Chem. 119, e25944 (2019), arXiv:1810.11653

${ }^{84}$ S. Lehtola, "Fully numerical Hartree-Fock and density functional calculations. I. Atoms," Int. J. Quantum Chem. 119, e25945 (2019), arXiv:1810.11651

${ }^{8}$ S. Lehtola, M. Dimitrova, and D. Sundholm, "Fully numerical electronic structure calculations on diatomic molecules in weak to strong magnetic fields," Mol. Phys. 118, e1597989 (2020) arXiv:1812.06274

${ }^{86}$ S. Lehtola, "Fully numerical calculations on atoms with fractional occupations and range-separated exchange functionals," Phys. Rev. A 101, 012516 (2020), arXiv:1908.02528

${ }^{87}$ P. Motamarri, S. Das, S. Rudraraju, K. Ghosh, D. Davydov, and V. Gavini, "DFT-FE - a massively parallel adaptive finiteelement code for large-scale density functional theory calculations," Comput. Phys. Commun. 246, 106853 (2020)

88"MADNESS - Multiresolution Adaptive Numerical Environment for Scientific Simulation,"

${ }^{89}$ G. te Velde, F. M. Bickelhaupt, E. J. Baerends, C. Fonseca Guerra, S. J. A. van Gisbergen, J. G. Snijders, and T. Ziegler, "Chemistry with ADF," J. Comput. Chem. 22, 931-967 (2001)

${ }^{90}$ G. M. J. Barca, C. Bertoni, L. Carrington, D. Datta, N. De Silva, J. E. Deustua, D. G. Fedorov, J. R. Gour, A. O. Gunina, E. Guidez, T. Harville, S. Irle, J. Ivanic, K. Kowalski, S. S. Leang, H. Li, W. Li, J. J. Lutz, I. Magoulas, J. Mato, V. Mironov, H. Nakata, B. Q. Pham, P. Piecuch, D. Poole, S. R. Pruitt, A. P. Rendell, L. B. Roskop, K. Ruedenberg, T. Sattasathuchana, M. W. Schmidt, J. Shen, L. Slipchenko, M. Sosonkina, V. Sundriyal, A. Tiwari, J. L. Galvez Vallejo, B. Westheimer, M. Włoch, P. Xu, F. Zahariev, and M. S. Gordon, "Recent developments in the general atomic and molecular electronic structure system," J. Chem. Phys. 152, 154102 (2020)

${ }^{91}$ H.-J. Werner, P. J. Knowles, F. R. Manby, J. A. Black, K. Doll, A. Heßelmann, D. Kats, A. Köhn, T. Korona, D. A. Kreplin, Q. Ma, T. F. Miller, A. Mitrushchenkov, K. A. Peterson, I. Polyak, G. Rauhut, and M. Sibaev, "The Molpro quantum chemistry package," J. Chem. Phys. 152, 144107 (2020)

${ }^{92}$ M. Kállay, P. R. Nagy, D. Mester, Z. Rolik, G. Samu, J. Csontos, J. Csóka, P. B. Szabó, L. Gyevi-Nagy, B. Hégely, I. Ladjánszki, L. Szegedy, B. Ladóczki, K. Petrov, M. Farkas, P. D. Mezei, and Á. Ganyecz, "The MRCC program system: Accurate quantum chemistry from water to proteins," J. Chem. Phys. 152, 074107 (2020)

${ }^{93}$ F. Neese, F. Wennmohs, U. Becker, and C. Riplinger, "The ORCA quantum chemistry program package," J. Chem. Phys. 152, 224108 (2020)

${ }^{94}$ S. G. Balasubramani, G. P. Chen, S. Coriani, M. Diedenhofen, M. S. Frank, Y. J. Franzke, F. Furche, R. Grotjahn, M. E. Harding, C. Hättig, A. Hellweg, B. Helmich-Paris, C. Holzer, U. Huniar, M. Kaupp, A. Marefat Khah, S. Karbalaei Khani, T. Müller, F. Mack, B. D. Nguyen, S. M. Parker, E. Perlt, D. Rappoport, K. Reiter, S. Roy, M. Rückert, G. Schmitz, M. Sierka, E. Tapavicza, D. P. Tew, C. van Wüllen, V. K. Voora, F. Weigend, A. Wodyński, and J. M. Yu, "TURBOMOLE: Modular program suite for ab initio quantum-chemical and condensed-matter simulations," J. Chem. Phys. 152, 184107 (2020).

${ }^{95}$ K. Lejaeghere, G. Bihlmayer, T. Bjorkman, P. Blaha, S. Blugel, V. Blum, D. Caliste, I. E. Castelli, S. J. Clark, A. D. Corso, S. de Gironcoli, T. Deutsch, J. K. Dewhurst, I. D. Marco, C. Draxl, M. D. ak, O. Eriksson, J. A. Flores-Livas, K. F. Garrity, L. Genovese, P. Giannozzi, M. Giantomassi, S. Goedecker, X. Gonze, O. Granas, E. K. U. Gross, A. Gulans, F. Gygi, D. R. Hamann, P. J. Hasnip, N. A. W. Holzwarth, D. I. an, D. B. Jochym, F. Jollet, D. Jones, G. Kresse, K. Koepernik, E. Kucukbenli, Y. O. Kvashnin, I. L. M. Locht, S. Lubeck, M. Marsman, N. Marzari, U. Nitzsche, L. Nordstrom, T. Ozaki, L. Paulatto, C. J. Pickard, W. Poelmans, M. I. J. Probert, K. Refson, M. Richter, G.-M. Rignanese, S. Saha, M. Scheffler, M. Schlipf, K. Schwarz, S. Sharma, F. Tavazza, P. Thunstrom, A. Tkatchenko, M. Torrent, D. Vanderbilt, M. J. van Setten, V. V. Speybroeck, J. M. Wills, J. R. Yates, G.-X. Zhang, and S. Cottenier, "Reproducibility in density functional theory calculations of solids," Science 351, aad3000-aad3000 (2016)

${ }^{96} \mathrm{~S}$. A. Ajila and D. Wu, "Empirical study of the effects of open source adoption on software development economics," J. Syst. Software 80, 1517-1529 (2007)

${ }^{9}$ M. J. T. Oliveira, N. Papior, Y. Pouillon, V. Blum, E. Artacho, D. Caliste, F. Corsetti, S. de Gironcoli, A. M. Elena, A. García, V. M. García-Suárez, L. Genovese, W. P. Huhn, G. Huhs, S. Kokott, E. Küçükbenli, A. H. Larsen, A. Lazzaro, I. V. Lebedeva, Y. Li, D. López-Durán, P. López-Tarifa, M. Lüders, M. A. L. Marques, J. Minar, S. Mohr, A. A. Mostofi, A. O'Cais, M. C. Payne, T. Ruh, D. G. A. Smith, J. M. Soler, D. A. Strubbe, N. Tancogne-Dejean, D. Tildesley, M. Torrent, and V. W.-z. Yu, "The CECAM electronic structure library and the modular software development paradigm," J. Chem. Phys. 153, 024117 (2020), arXiv:2005.05756

${ }^{98}$ E. Caldeweyher, C. Bannwarth, and S. Grimme, "Extension of the D3 dispersion coefficient model," J. Chem. Phys. 147, $034112(2017)$

${ }^{9 y}$ E. Caldeweyher, S. Ehlert, A. Hansen, H. Neugebauer, S. Spicher, C. Bannwarth, and S. Grimme, "A generally applicable atomic-charge dependent london dispersion correction," J. Chem. Phys. 150, 154122 (2019)

${ }^{100}$ E. Caldeweyher, J.-M. Mewes, S. Ehlert, and S. Grimme, "Extension and evaluation of the $\mathrm{d} 4$ london-dispersion model for periodic systems," Phys. Chem. Chem. Phys. 22, 8499-8512 (2020).

${ }^{10}$ W. L. DeLano, "The case for open-source software in drug discovery," Drug Discovery Today 10, 213-217 (2005)

${ }^{102}$ D. G. A. Smith, L. A. Burns, D. A. Sirianni, D. R. Nascimento, A. Kumar, A. M. James, J. B. Schriber, T. Zhang, B. Zhang, A. S. Abbott, E. J. Berquist, M. H. Lechner, L. A. Cunha, A. G. Heide, J. M. Waldrop, T. Y. Takeshita, A. Alenaizan, D. Neuhauser, R. A. King, A. C. Simmonett, J. M. Turney, H. F. Schaefer, F. A. Evangelista, A. E. DePrince, T. D. Crawford, K. Patkowski, and C. D. Sherrill, "PSI4NumPy: An Interactive Quantum Chemistry Programming Environment for Reference Implementations and Rapid Development," J. Chem. Theory Comput. 14, 3504-3511 (2018)

${ }^{103}$ S. Lehtola, F. Blockhuys, and C. Van Alsenoy, "An Overview of Self-Consistent Field Calculations Within Finite Basis Sets," Molecules 25, 1218 (2020) arXiv:1912.12029

${ }^{104}$ G. Samsonidze and B. Kozinsky, "Half-heusler compounds for use in thermoelectric generators," (U.S. Patent 20170141282, May 2017).

${ }^{105}$ J.-H. Ye and C.-L. Huang, "Method for crystallizing metal oxide semiconductor layer, semiconductor structure, active array substrate, and indium gallium zinc oxide crystal," (U.S. Patent 20180166474, June 2018).

${ }^{106}$ E. Strohmaier, H. W. Meuer, J. Dongarra, and H. D. Simon, "The TOP500 list and progress in high-performance computing," Computer 48, 42-49 (2015) 
${ }^{107}$ H. W. Meuer, E. Strohmaier, J. Dongarra, H. Simon, and M. Meuer, "Top500," https://top500.org/, accessed Thu May 202021.

${ }^{108}$ A. Szabo and N. S. Ostlund, Modern quantum chemistry: introduction to advanced electronic structure theory (Dover Pubns, 1996).

${ }^{109}$ W. J. Geldenhuys, K. E. Gaasch, M. Watson, D. D. Allen, and C. J. V. der Schyf, "Optimizing the use of open-source software applications in drug discovery," Drug Discovery Today 11, 127$132(2006)$

${ }^{110}$ S. Pirhadi, J. Sunseri, and D. R. Koes, "Open source molecular modeling," J. Mol. Graph. Model. 69, 127-143 (2016).

${ }^{111}$ J. Rodríguez-Becerra, L. Cáceres-Jensen, T. Díaz, S. Druker, V. B. Padilla, J. Pernaa, and M. Aksela, "Developing technological pedagogical science knowledge through educational computational chemistry: a case study of pre-service chemistry teachers' perceptions," Chem. Educ. Res. Pract. 21, 638-654 (2020)

${ }^{112}$ L. Talirz, L. M. Ghiringhelli, and B. Smit, "Trends in atomistic simulation software usage," (2021), arXiv:2108.12350 [condmat.mtrl-sci]

113"Python package index - pypi," https://pypi.org/, accessed 7 July 2021.

${ }^{114}$ Continuum Analytics, "Conda package manager," https://conda.io/, accessed 26 May 2021.

${ }^{115}$ P. Hohenberg and W. Kohn, "Inhomogeneous Electron Gas," Phys. Rev. 136, B864-B871 (1964)

${ }^{116}$ W. Kohn and L. J. Sham, "Self-Consistent Equations Including Exchange and Correlation Effects," Phys. Rev. 140, A1133A1138 (1965)

${ }^{11 '}$ S. F. Boys, "Electronic Wave Functions. I. A General Method of Calculation for the Stationary States of Any Molecular System," Proc. R. Soc. A Math. Phys. Eng. Sci. 200, 542-554 (1950)

${ }^{118}$ L. E. McMurchie and E. R. Davidson, "One- and two-electron integrals over cartesian Gaussian functions," J. Comput. Phys. 26, 218-231 (1978)

${ }^{119} \mathrm{~S}$. Obara and A. Saika, "Efficient recursive computation of molecular integrals over Cartesian Gaussian functions," J. Chem. Phys. 84, 3963 (1986)

${ }^{120}$ E. R. Davidson and D. Feller, "Basis set selection for molecular calculations," Chem. Rev. 86, 681-696 (1986)

${ }^{121}$ J. G. Hill, "Gaussian basis sets for molecular applications," Int. J. Quantum Chem. 113, 21-34 (2013)

${ }^{122}$ F. Jensen, "Atomic orbital basis sets," Wiley Interdiscip. Rev. Comput. Mol. Sci. 3, 273-295 (2013).

${ }^{125}$ T. Shiozaki, "BAGEL: Brilliantly Advanced General Electronicstructure Library," Wiley Interdiscip. Rev. Comput. Mol. Sci. 8, e1331 (2018) arXiv:1707.03771

${ }^{124}$ D. B. Williams-Young, A. Petrone, S. Sun, T. F. Stetina, P. Lestrange, C. E. Hoyer, D. R. Nascimento, L. Koulias, A. Wildman, J. Kasper, J. J. Goings, F. Ding, A. E. DePrince, E. F. Valeev, and X. Li, "The chronus quantum software package," WIREs Comput. Mol. Sci. 10, e1436 (2020)

${ }^{125}$ K. Aidas, C. Angeli, K. L. Bak, V. Bakken, R. Bast, L. Boman, O. Christiansen, R. Cimiraglia, S. Coriani, P. Dahle, E. K. Dalskov, U. Ekström, T. Enevoldsen, J. J. Eriksen, P. Ettenhuber, B. Fernández, L. Ferrighi, H. Fliegl, L. Frediani, K. Hald, A. Halkier, C. Hättig, H. Heiberg, T. Helgaker, A. C. Hennum, H. Hettema, E. Hjertenaes, S. Høst, I.-M. Høyvik, M. F. Iozzi, B. Jansík, H. J. A. Jensen, D. Jonsson, P. Jørgensen, J. Kauczor, S. Kirpekar, T. Kjaergaard, W. Klopper, S. Knecht, R. Kobayashi, H. Koch, J. Kongsted, A. Krapp, K. Kristensen, A. Ligabue, O. B. Lutnaes, J. I. Melo, K. V. Mikkelsen, R. H. Myhre, C. Neiss, C. B. Nielsen, P. Norman, J. Olsen, J. M. H. Olsen, A. Osted, M. J. Packer, F. Pawlowski, T. B. Pedersen, P. F. Provasi, S. Reine, Z. Rinkevicius, T. A. Ruden, K. Ruud, V. V. Rybkin, P. Sałek, C. C. M. Samson, A. S. de Merás, T. Saue, S. P. A. Sauer, B. Schimmelpfennig, K. Sneskov, A. H. Steindal, K. O. Sylvester-Hvid, P. R. Taylor, A. M. Teale, E. I. Tellgren, D. P. Tew, A. J. Thorvaldsen, L. Thøgersen, O. Vahtras, M. A. Watson, D. J. D. Wilson, M. Ziolkowski, and
H. Ågren, "The Dalton quantum chemistry program system," Wiley Interdiscip. Rev. Comput. Mol. Sci. 4, 269-284 (2014),

${ }^{126}$ E. Rudberg, E. H. Rubensson, P. Sałek, and A. Kruchinina, "Ergo: An open-source program for linear-scaling electronic structure calculations," SoftwareX 7, 107-111 (2018)

${ }^{127}$ S. D. Folkestad, E. F. Kjønstad, R. H. Myhre, J. H. Andersen, A. Balbi, S. Coriani, T. Giovannini, L. Goletto, T. S. Haugland, A. Hutcheson, I.-M. Høyvik, T. Moitra, A. C. Paul, M. Scavino, A. S. Skeidsvoll, A. H. Tveten, and H. Koch, " $e^{T}$ 1.0: An open source electronic structure program with emphasis on coupled cluster and multilevel methods," J. Chem. Phys. 152, 184103 (2020), arXiv:2002.05631

${ }^{128}$ D. Poole, J. L. G. Vallejo, and M. S. Gordon, "A new kid on the block: Application of julia to hartree-fock calculations," J. Chem. Theory Comput. 16, 5006-5013 (2020)

${ }^{129}$ F. Bruneval, T. Rangel, S. M. Hamed, M. Shao, C. Yang, and J. B. Neaton, "molgw 1: Many-body perturbation theory software for atoms, molecules, and clusters," Comput. Phys. Commun. 208, 149-161 (2016)

${ }^{130}$ C. Peng, C. A. Lewis, X. Wang, M. C. Clement, K. Pierce, V. Rishi, F. Pavošević, S. Slattery, J. Zhang, N. Teke, A. Kumar, C. Masteran, A. Asadchev, J. A. Calvin, and E. F. Valeev, "Massively Parallel Quantum Chemistry: A high-performance research platform for electronic structure," J. Chem. Phys. 153, $044120(2020)$

${ }^{131}$ R. P. Mueller, "PyQuante: Python quantum chemistry," http://pyquante.sourceforge.net/, accessed 6 July 2021.

${ }^{132}$ J. P. Unsleber, T. Dresselhaus, K. Klahr, D. Schnieders, M. Böckers, D. Barton, and J. Neugebauer, "Serenity: A subsystem quantum chemistry program," J. Comput. Chem. 39, 788-798 (2018)

${ }^{133}$ E. Kjellgren, "SlowQuant," https://github.com/erikkjellgren/SlowQuant, accessed 6 July 2021.

${ }^{134}$ Z. Rinkevicius, X. Li, O. Vahtras, K. Ahmadzadeh, M. Brand, M. Ringholm, N. H. List, M. Scheurer, M. Scott, A. Dreuw, and P. Norman, "VeloxChem: A python-driven densityfunctional theory program for spectroscopy simulations in highperformance computing environments," WIREs Comput. Mol. Sci. 10, e1457 (2019)

${ }^{135} \mathrm{P}$. Souvatzis, "Uquantchem: A versatile and easy to use quantum chemistry computational software," Computer Physics Communications 185, 415-421 (2014)

${ }^{130} \mathrm{~F}$. Bloch, "Bemerkung zur Elektronentheorie des Ferromagnetismus und der elektrischen Leitfähigkeit," Z. Phys. 57, 545555 (1929).

${ }^{137}$ P. Kratzer and J. Neugebauer, "The Basics of Electronic Structure Theory for Periodic Systems," Front. Chem. 7, 1-18 (2019)

${ }^{138} \mathrm{P}$. Schwerdtfeger, "The Pseudopotential Approximation in Electronic Structure Theory," ChemPhysChem 12, 3143-3155 (2011)

${ }^{15 y}$ A. H. Romero, D. C. Allan, B. Amadon, G. Antonius, T. Applencourt, L. Baguet, J. Bieder, F. Bottin, J. Bouchet, E. Bousquet, F. Bruneval, G. Brunin, D. Caliste, M. Côté, J. Denier, C. Dreyer, P. Ghosez, M. Giantomassi, Y. Gillet, O. Gingras, D. R. Hamann, G. Hautier, F. Jollet, G. Jomard, A. Martin, H. P. C. Miranda, F. Naccarato, G. Petretto, N. A. Pike, V. Planes, S. Prokhorenko, T. Rangel, F. Ricci, G.-M. Rignanese, M. Royo, M. Stengel, M. Torrent, M. J. van Setten, B. Van Troeye, M. J. Verstraete, J. Wiktor, J. W. Zwanziger, and X. Gonze, "ABINIT: Overview and focus on selected capabilities," J. Chem. Phys. 152, 124102 (2020)

${ }^{140}$ S. Kang, J. Woo, J. Kim, H. Kim, Y. Kim, J. Lim, S. Choi, and W. Y. Kim, "ACE-Molecule: An open-source real-space quantum chemistry package," J. Chem. Phys. 152, 124110 (2020)

${ }^{141}$ L. E. Ratcliff, W. Dawson, G. Fisicaro, D. Caliste, S. Mohr, A. Degomme, B. Videau, V. Cristiglio, M. Stella, M. D'Alessandro, S. Goedecker, T. Nakajima, T. Deutsch, and L. Genovese, "Flexibilities of wavelets as a computational basis set for large-scale electronic structure calculations," J. Chem. Phys. 152, 194110 (2020) 
${ }^{142}$ A. Nakata, J. S. Baker, S. Y. Mujahed, J. T. L. Poulton, S. Arapan, J. Lin, Z. Raza, S. Yadav, L. Truflandier, T. Miyazaki, and D. R. Bowler, "Large scale and linear scaling DFT with the CONQUEST code," J. Chem. Phys. 152, 164112 (2020) arXiv:2002.07704

${ }^{143}$ T. D. Kühne, M. Iannuzzi, M. Del Ben, V. V. Rybkin, P. Seewald, F. Stein, T. Laino, R. Z. Khaliullin, O. Schütt, F. Schiffmann, D. Golze, J. Wilhelm, S. Chulkov, M. H. BaniHashemian, V. Weber, U. Borštnik, M. Taillefumier, A. S. Jakobovits, A. Lazzaro, H. Pabst, T. Müller, R. Schade, M. Guidon, S. Andermatt, N. Holmberg, G. K. Schenter, A. Hehn, A. Bussy, F. Belleflamme, G. Tabacchi, A. Glöß, M. Lass, I. Bethune, C. J. Mundy, C. Plessl, M. Watkins, J. VandeVondele, M. Krack, and J. Hutter, "CP2K: An electronic structure and molecular dynamics software package - Quickstep: Efficient and accurate electronic structure calculations," J. Chem. Phys. 152, 194103 (2020) arXiv:2003.03868

${ }^{144}$ M. F. Herbst, A. Levitt, and E. Cancès, "DFTK: A julian approach for simulating electrons in solids," JuliaCon Proceedings 3, 69 (2021)

145"The Elk Code," http://elk.sourceforge.net/

${ }^{146}$ A. Gulans, S. Kontur, C. Meisenbichler, D. Nabok, P. Pavone, S. Rigamonti, S. Sagmeister, U. Werner, and C. Draxl, "exciting: a full-potential all-electron package implementing densityfunctional theory and many-body perturbation theory," J. Phys. Condens. Matter 26, 363202 (2014)

14r"FLEUR," http://www.flapw.de

${ }^{148}$ J. Enkovaara, C. Rostgaard, J. J. Mortensen, J. Chen, M. Dułak, L. Ferrighi, J. Gavnholt, C. Glinsvad, V. Haikola, H. A. Hansen, H. H. Kristoffersen, M. Kuisma, A. H. Larsen, L. Lehtovaara, M. Ljungberg, O. Lopez-Acevedo, P. G. Moses, J. Ojanen, T. Olsen, V. Petzold, N. A. Romero, J. StausholmMøller, M. Strange, G. A. Tritsaris, M. Vanin, M. Walter, B. Hammer, H. Häkkinen, G. K. H. Madsen, R. M. Nieminen, J. K. Nørskov, M. Puska, T. T. Rantala, J. Schiøtz, K. S. Thygesen, and K. W. Jacobsen, "Electronic structure calculations with GPAW: a real-space implementation of the projector augmented-wave method." J. Phys. Condens. Matter 22, 253202 (2010)

${ }^{149}$ R. Sundararaman, K. Letchworth-Weaver, K. A. Schwarz, D. Gunceler, Y. Ozhabes, and T. A. Arias, "JDFTx: Software for joint density-functional theory," SoftwareX 6, 278-284 (2017)

${ }^{150}$ Q. Xu, A. Sharma, and P. Suryanarayana, "M-SPARC: Matlabsimulation package for ab-initio real-space calculations," SoftwareX 11, 100423 (2020).

${ }^{151}$ N. Tancogne-Dejean, M. J. T. Oliveira, X. Andrade, H. Appel, C. H. Borca, G. Le Breton, F. Buchholz, A. Castro, S. Corni, A. A. Correa, U. De Giovannini, A. Delgado, F. G. Eich, J. Flick, G. Gil, A. Gomez, N. Helbig, H. Hübener, R. Jestädt, J. JornetSomoza, A. H. Larsen, I. V. Lebedeva, M. Lüders, M. A. L. Marques, S. T. Ohlmann, S. Pipolo, M. Rampp, C. A. Rozzi, D. A. Strubbe, S. A. Sato, C. Schäfer, I. Theophilou, A. Welden, and A. Rubio, "Octopus, a computational framework for exploring light-driven phenomena and quantum dynamics in extended and finite systems," J. Chem. Phys. 152, 124119 (2020) arXiv:1912.07921

${ }^{152}$ T. Ozaki and H. Kino, "Numerical atomic basis orbitals from $\mathrm{H}$ to Kr," Phys. Rev. B 69, 195113 (2004)

${ }^{153}$ Y. Saad, J. R. Chelikowsky, and S. M. Shontz, "Numerical Methods for Electronic Structure Calculations of Materials," SIAM Rev. 52, 3-54 (2010)

${ }^{154}$ F. Fathurrahman, M. K. Agusta, A. G. Saputro, and H. K. Dipojono, "PWDFT.jl: A julia package for electronic structure calculation using density functional theory and plane wave basis," Comput. Phys. Commun. 256, 107372 (2020).

${ }^{155}$ E. L. Briggs, D. J. Sullivan, and J. Bernholc, "Real-space multigrid-based approach to large-scale electronic structure calculations," Phys. Rev. B 54, 14362-14375 (1996)

${ }^{156}$ A. García, N. Papior, A. Akhtar, E. Artacho, V. Blum, E. Bosoni, P. Brandimarte, M. Brandbyge, J. I. Cerdá,
F. Corsetti, R. Cuadrado, V. Dikan, J. Ferrer, J. Gale, P. GarcíaFernández, V. M. García-Suárez, S. García, G. Huhs, S. Illera, R. Korytár, P. Koval, I. Lebedeva, L. Lin, P. López-Tarifa, S. G. Mayo, S. Mohr, P. Ordejón, A. Postnikov, Y. Pouillon, M. Pruneda, R. Robles, D. Sánchez-Portal, J. M. Soler, R. Ullah, V. W.-z. Yu, and J. Junquera, "Siesta: Recent developments and applications," J. Chem. Phys. 152, 204108 (2020)

${ }^{157}$ F. Gygi, "Architecture of qbox: A scalable first-principles molecular dynamics code," IBM J. Res. Dev. 52, 137-144 (2008)

${ }^{158}$ Q. Xu, A. Sharma, B. Comer, H. Huang, E. Chow, A. J. Medford, J. E. Pask, and P. Suryanarayana, "SPARC: Simulation package for ab-initio real-space calculations," SoftwareX 15, 100709 (2021)

${ }^{159}$ S. Lehtola, "A review on non-relativistic, fully numerical electronic structure calculations on atoms and diatomic molecules," Int. J. Quantum Chem. 119, e25968 (2019) arXiv:1902.01431

${ }^{160}$ S. R. Jensen, T. Flå, D. Jonsson, R. S. Monstad, K. Ruud, and L. Frediani, "Magnetic properties with multiwavelets and DFT: the complete basis set limit achieved," Phys. Chem. Chem. Phys. 18, 21145-21161 (2016)

${ }^{101}$ R. J. Harrison, G. Beylkin, F. A. Bischoff, J. A. Calvin, G. I. Fann, J. Fosso-Tande, D. Galindo, J. R. Hammond, R. Hartman-Baker, J. C. Hill, J. Jia, J. S. Kottmann, M.J. Y. Ou, J. Pei, L. E. Ratcliff, M. G. Reuter, A. C. RichieHalford, N. A. Romero, H. Sekino, W. A. Shelton, B. E. Sundahl, W. S. Thornton, E. F. Valeev, Á. Vázquez-Mayagoitia, N. Vence, T. Yanai, and Y. Yokoi, "MADNESS: A multiresolution, adaptive numerical environment for scientific simulation," SIAM J. Sci. Comput. 38, S123-S142 (2016).

${ }^{162} \mathrm{~J}$. Kobus, "A finite difference Hartree-Fock program for atoms and diatomic molecules," Comput. Phys. Commun. 184, 799$811(2013)$

${ }^{103}$ P. Koskinen and V. Mäkinen, "Density-functional tight-binding for beginners," Comput. Mater. Sci. 47, 237-253 (2009).

${ }^{164} \mathrm{G}$. Seifert and J.-O. Joswig, "Density-functional tight bindingan approximate density-functional theory method," Wiley Interdiscip. Rev.: Comput. Mol. Sci. 2, 456-465 (2012)

${ }^{105}$ M. Gaus, Q. Cui, and M. Elstner, "Density functional tight binding: application to organic and biological molecules," Wiley Interdiscip. Rev.: Comput. Mol. Sci. 4, 49-61 (2013).

${ }^{106}$ W. Thiel, "Semiempirical quantum-chemical methods," Wiley Interdiscip. Rev. Comput. Mol. Sci. 4, 145-157 (2014)

${ }^{10}{ }^{\prime}$ C. Bannwarth, E. Caldeweyher, S. Ehlert, A. Hansen, P. Pracht, J. Seibert, S. Spicher, and S. Grimme, "Extended tight-binding quantum chemistry methods," WIREs Comput. Mol. Sci. 11, e1493 (2021)

${ }^{108}$ B. Hourahine, B. Aradi, V. Blum, F. Bonafé, A. Buccheri, C. Camacho, C. Cevallos, M. Y. Deshaye, T. Dumitrică, A. Dominguez, S. Ehlert, M. Elstner, T. van der Heide, J. Hermann, S. Irle, J. J. Kranz, C. Köhler, T. Kowalczyk, T. Kubař, I. S. Lee, V. Lutsker, R. J. Maurer, S. K. Min, I. Mitchell, C. Negre, T. A. Niehaus, A. M. N. Niklasson, A. J. Page, A. Pecchia, G. Penazzi, M. P. Persson, J. Řezáč, C. G. Sánchez, M. Sternberg, M. Stöhr, F. Stuckenberg, A. Tkatchenko, V. W. Yu, and T. Frauenheim, "DFTB+, a software package for efficient approximate density functional theory based atomistic simulations," J. Chem. Phys. 152, 124101 (2020)

${ }^{169}$ N. Bock, M. J. Cawkwell, J. D. Coe, A. Krishnapriyan, M. P. Kroonblawd, A. Lang, , C. Liu, E. M. Saez, S. M. Mniszewski, C. F. A. Negre, A. M. N. Niklasson, E. Sanville, M. A. Wood, and P. Yang, "Latte," https://github.com/lanl/LATTE (2008), accessed 12 July 2021.

${ }^{170} \mathrm{~T}$. Husch and M. Reiher, "Comprehensive Analysis of the Neglect of Diatomic Differential Overlap Approximation," J. Chem. Theory Comput. 14, 5169-5179 (2018). arXiv:1806.05615

171"Jmol: an open-source Java viewer for chemical structures in 3D. http://www.jmol.org,".

${ }^{172}$ M. Hanwell, D. Curtis, D. Lonie, T. Vandermeerschd, E. Zurek, and G. Hutchison, "Avogadro: An advanced semantic chemical 
editor, visualization, and analysis platform," Journal of Cheminformatics 4 (2012), 10.1186/1758-2946-4-17

${ }^{173}$ A. Gilbert, "Iqmol, a free open-source molecular editor and visualization package." http://iqmol.org, accessed June 262021.

${ }^{174}$ Schrödinger Inc., "Pymol, a molecular visualization system," https://github.com/schrodinger/pymol-open-source, accessed 6 July 2021.

${ }^{175}$ A. H. Larsen, J. J. Mortensen, J. Blomqvist, I. E. Castelli, R. Christensen, M. Dułak, J. Friis, M. N. Groves, B. Hammer, C. Hargus, E. D. Hermes, P. C. Jennings, P. B. Jensen, J. Kermode, J. R. Kitchin, E. L. Kolsbjerg, J. Kubal, K. Kaasbjerg, S. Lysgaard, J. B. Maronsson, T. Maxson, T. Olsen, L. Pastewka, A. Peterson, C. Rostgaard, J. Schiøtz, O. Schütt, M. Strange, K. S. Thygesen, T. Vegge, L. Vilhelmsen, M. Walter, Z. Zeng, and K. W. Jacobsen, "The atomic simulation environment - a python library for working with atoms," J. Phys.: Condens. Matter 29, 273002 (2017)

${ }^{\text {Tro }}$ S. Kim, J. Chen, T. Cheng, A. Gindulyte, J. He, S. He, Q. Li, B. A. Shoemaker, P. A. Thiessen, B. Yu, L. Zaslavsky, J. Zhang, and E. E. Bolton, "PubChem in 2021: new data content and improved web interfaces," Nucleic Acids Research 49, D1388D1395 (2020)

${ }^{1 r}$ N. M. O'Boyle, M. Banck, C. A. James, C. Morley, T. Vandermeersch, and G. R. Hutchison, "Open Babel: An open chemical toolbox," J. Cheminf. 3, 33 (2011)

178 N. M. O'Boyle, A. L. Tenderholt, and K. M. Langner, "cclib: A library for package-independent computational chemistry algorithms," J. Comput. Chem. 29, 839-845 (2008)

${ }^{179}$ T. Lu and F. Chen, "Multiwfn: A multifunctional wavefunction analyzer," J. Comput. Chem. 33, 580-592 (2011)

${ }^{180}$ G. Hermann, V. Pohl, J. C. Tremblay, B. Paulus, H.-C. Hege, and A. Schild, "ORBKIT: A modular python toolbox for cross-platform postprocessing of quantum chemical wavefunction data," J. Comput. Chem. 37, 1511-1520 (2016).

${ }^{181} \mathrm{~S}$. Lehtola and A. J. Karttunen, "git repository containing a copy of the supporting information," https://github.com/susilehtola/fosschemistry, accessed 8 August 2021.

${ }^{182}$ C. Bannwarth, S. Ehlert, and S. Grimme, "GFN2-xTB-an accurate and broadly parametrized self-consistent tight-binding quantum chemical method with multipole electrostatics and density-dependent dispersion contributions," J. Chem. Theory Comput. 15, 1652-1671 (2019)

${ }^{183}$ J. P. Menzel, M. Kloppenburg, J. Belić, H. J. M. Groot, L. Visscher, and F. Buda, "Efficient workflow for the investigation of the catalytic cycle of water oxidation catalysts: Combining GFN-xTB and density functional theory," J. Comput. Chem. (2021), 10.1002/jcc.26721

${ }^{184}$ N. Tasinato, C. Puzzarini, and V. Barone, "Correct modeling of cisplatin: a paradigmatic case," Angew. Chem. - Int. Ed. 56, 13838-13841 (2017)

${ }^{185} \mathrm{~W}$. Liu and R. Franke, "Comprehensive relativistic ab initio and density functional theory studies on $\mathrm{PtH}, \mathrm{PtF}, \mathrm{PtCl}$, and $\mathrm{Pt}\left(\mathrm{NH}_{3}\right)_{2} \mathrm{Cl}_{2}$," J. Comput. Chem. 23, 564-575 (2002).

${ }^{186}$ A. D. Becke, "Density-functional exchange-energy approximation with correct asymptotic behavior," Phys. Rev. A 38, 30983100 (1988)

${ }^{18}$ J. P. Perdew, "Density-functional approximation for the correlation energy of the inhomogeneous electron gas," Phys. Rev. B 33, 8822-8824 (1986)

${ }^{188} \mathrm{C}$. Adamo and V. Barone, "Toward reliable density functional methods without adjustable parameters: The PBE0 model," J. Chem. Phys. 110, 6158-6170 (1999)

${ }^{189}$ M. Ernzerhof and G. E. Scuseria, "Assessment of the PerdewBurke-Ernzerhof exchange-correlation functional," J. Chem. Phys. 110, 5029-5036 (1999)

${ }^{190}$ V. Vetere, C. Adamo, and P. Maldivi, "Performance of the 'parameter free' PBE0 functional for the modeling of molecular properties of heavy metals," Chem. Phys. Lett. 325, 99-105 (2000)
${ }^{191}$ M. Bühl, C. Reimann, D. A. Pantazis, T. Bredow, and F. Neese, "Geometries of Third-Row Transition-Metal Complexes from Density-Functional Theory," J. Chem. Theory Comput. 4, 14491459 (2008)

${ }^{192}$ W. J. Hehre, R. F. Stewart, and J. A. Pople, "Self-Consistent Molecular-Orbital Methods. I. Use of Gaussian Expansions of Slater-Type Atomic Orbitals," J. Chem. Phys. 51, 2657 (1969)

${ }^{193}$ J. S. Binkley, J. A. Pople, and W. J. Hehre, "Self-consistent molecular orbital methods. 21. Small split-valence basis sets for first-row elements," J. Am. Chem. Soc. 102, 939 (1980)

${ }^{194}$ W. J. Hehre, R. Ditchfield, and J. A. Pople, "Self-Consistent Molecular Orbital Methods. XII. Further Extensions of Gaussian-Type Basis Sets for Use in Molecular Orbital Studies of Organic Molecules," J. Chem. Phys. 56, 2257-2261 (1972)

${ }^{195}$ B. P. Pritchard, D. Altarawy, B. Didier, T. D. Gibson, and T. L. Windus, "New Basis Set Exchange: An Open, Up-to-Date Resource for the Molecular Sciences Community," J. Chem. Inf. Model. 59, 4814-4820 (2019)

${ }^{196} \mathrm{~F}$. Weigend and R. Ahlrichs, "Balanced basis sets of split valence, triple zeta valence and quadruple zeta valence quality for H to Rn: Design and assessment of accuracy." Phys. Chem. Chem. Phys. 7, 3297-305 (2005)

${ }^{19}$ 'P. Pyykkö, "Relativistic effects in chemistry: more common than you thought." Annu. Rev. Phys. Chem. 63, 45-64 (2012).

${ }^{198}$ P. Pyykkö, "The physics behind chemistry and the periodic table." Chem. Rev. 112, 371-84 (2012)

${ }^{199}$ M. Dolg, "Chapter 14 relativistic effective core potentials," in Theoretical and Computational Chemistry (Elsevier, 2002) pp. 793-862.

${ }^{200}$ D. Rappoport and F. Furche, "Property-optimized gaussian basis sets for molecular response calculations." J. Chem. Phys. 133, $134105(2010)$

${ }^{201}$ S. Ehlert, U. Huniar, J. Ning, J. W. Furness, J. Sun, A. D. Kaplan, J. P. Perdew, and J. G. Brandenburg, " $\mathrm{r}^{2}$ scan-d4: Dispersion corrected meta-generalized gradient approximation for general chemical applications," J. Chem. Phys. 154, 061101 (2021)

${ }^{202}$ N. Tasinato, C. Puzzarini, and V. Barone, "Correct modeling of cisplatin: a paradigmatic case," Angewandte Chemie International Edition 56, 13838-13841 (2017)

${ }^{203}$ C. Møller and M. S. M. Plesset, "Note on an Approximation Treatment for Many-Electron Systems," Phys. Rev. 46, 618-622 (1934)

${ }^{204}$ J. Cížek, "On the Correlation Problem in Atomic and Molecular Systems. Calculation of Wavefunction Components in UrsellType Expansion Using Quantum-Field Theoretical Methods," J. Chem. Phys. 45, 4256-4266 (1966)

${ }^{205}$ S. Grimme, "Exploration of Chemical Compound, Conformer, and Reaction Space with Meta-Dynamics Simulations Based on Tight-Binding Quantum Chemical Calculations," J. Chem. Theory Comput. 15, 2847-2862 (2019).

${ }^{200}$ P. Pracht, F. Bohle, and S. Grimme, "Automated exploration of the low-energy chemical space with fast quantum chemical methods," Phys. Chem. Chem. Phys. 22, 7169-7192 (2020)

${ }^{207}$ P. Pracht and S. Grimme, "Calculation of absolute molecular entropies and heat capacities made simple," Chem. Sci. 12, 6551$6568(2021)$

${ }^{208}$ J. L. Whitten, "Coulombic potential energy integrals and approximations," J. Chem. Phys. 58, 4496 (1973)

${ }^{209}$ E. J. Baerends, D. E. Ellis, and P. Ros, "Self-consistent molecular Hartree-Fock-Slater calculations I. The computational procedure," Chem. Phys. 2, 41-51 (1973)

${ }^{210}$ B. I. Dunlap, J. W. D. Connolly, and J. R. Sabin, "On the applicability of LCAO-X $\alpha$ methods to molecules containing transition metal atoms: The nickel atom and nickel hydride," Int. J. Quantum Chem. 12, 81-87 (1977)

${ }^{211}$ B. I. Dunlap, J. W. D. Connolly, and J. R. Sabin, "On some approximations in applications of X $\alpha$ theory," J. Chem. Phys. 71, 3396 (1979)

${ }^{212}$ B. I. Dunlap, N. Rösch, and S. B. Trickey, "Variational fitting methods for electronic structure calculations," Mol. Phys. 108, 
$3167-3180(2010)$

${ }^{113} \mathrm{~F}$. Weigend, "Hartree-Fock exchange fitting basis sets for $\mathrm{H}$ to Rn," J. Comput. Chem. 29, 167-175 (2008).

${ }^{214}$ T. H. Dunning, "Gaussian basis sets for use in correlated molecular calculations. I. The atoms boron through neon and hydrogen," J. Chem. Phys. 90, 1007 (1989)

${ }^{215}$ E. Van Lenthe and E. J. Baerends, "Optimized Slater-type basis sets for the elements 1-118." J. Comput. Chem. 24, 1142-56 (2003)

${ }^{216}$ D. P. Chong, E. van Lenthe, S. Van Gisbergen, and E. J. Baerends, "Even-tempered Slater-type orbitals revisited: from hydrogen to krypton." J. Comput. Chem. 25, 1030-6 (2004).

${ }^{217}$ M. Bühl and H. Kabrede, "Geometries of Transition-Metal Complexes from Density-Functional Theory," J. Chem. Theory Comput. 2, 1282-1290 (2006)

${ }^{218}$ S. Grimme, C. Bannwarth, and P. Shushkov, "A Robust and Accurate Tight-Binding Quantum Chemical Method for Structures, Vibrational Frequencies, and Noncovalent Interactions of Large Molecular Systems Parametrized for All spd-Block Elements $(Z=1-86)$," J. Chem. Theory Comput. 13, 1989-2009 (2017)

${ }^{219}$ P. A. M. Dirac, "Note on Exchange Phenomena in the Thomas Atom," Math. Proc. Cambridge Philos. Soc. 26, 376-385 (1930)

${ }^{220}$ J. P. Perdew and Y. Wang, "Accurate and simple analytic representation of the electron-gas correlation energy," Phys. Rev. B 45, 13244-13249 (1992)

${ }^{221}$ J. W. Furness, A. D. Kaplan, J. Ning, J. P. Perdew, and J. Sun, "Accurate and numerically efficient $\mathrm{r}^{2}$ SCAN meta-generalized gradient approximation," J. Phys. Chem. Lett. 11, 8208-8215 (2020)

${ }^{222}$ J. W. Furness, A. D. Kaplan, J. Ning, J. P. Perdew, and J. Sun, "Correction to "accurate and numerically efficient $\mathrm{r}^{2} \mathrm{SCAN}$ meta-generalized gradient approximation"," J. Phys. Chem. Lett. 11, 9248-9248 (2020)

${ }^{223} \mathrm{~F}$. Weigend, "Accurate Coulomb-fitting basis sets for H to Rn." Phys. Chem. Chem. Phys. 8, 1057-65 (2006)

${ }^{224}$ W. J. Pietro and W. J. Hehre, "Molecular orbital theory of the properties of inorganic and organometallic compounds. 3.STO$3 \mathrm{~g}$ basis sets for first- and second-row transition metals," J. Comput. Chem. 4, 241-251 (1983)

${ }^{225}$ R. J. French, L. Hedberg, K. Hedberg, G. L. Gard, and B. M. Johnson, "Molecular structure and quadratic force field of chromyl fluoride, $\mathrm{CrO}_{2} \mathrm{~F}_{2}$," Inorg. Chem. 22, 892-895 (1983).

${ }^{226}$ W. L. Levason, J. S. Ogden, A. K. Saad, N. A. Young, A. K. Brisdon, P. J. Holliman, J. H. Holloway, and E. G. Hope, "Metal $k$-edge EXAFS (extended x-ray absorption fine structure) studies of $\mathrm{CrO}_{2} \mathrm{~F}_{2}$ and $\mathrm{MnO}_{3} \mathrm{~F}$ at 10K," J. Fluorine Chem. 53, 43-51 (1991)

${ }^{22}$ B. Gilbert, B. H. Frazer, H. Zhang, F. Huang, J. F. Banfield, D. Haskel, J. C. Lang, G. Srajer, and G. D. Stasio, "X-ray absorption spectroscopy of the cubic and hexagonal polytypes of zinc sulfide," Phys. Rev. B 66, 245205 (2002)

${ }^{228}$ P. J. Gardner and P. Pang, "Thermodynamics of the zinc sulphide transformation, sphalerite $\rightarrow$ wurtzite, by modified entrainment," J. Chem. Soc., Faraday Trans. 1 84, 1879 (1988).

${ }^{229}$ K. F. Garrity, J. W. Bennett, K. M. Rabe, and D. Vanderbilt, "Pseudopotentials for high-throughput DFT calculations," Comput. Mater. Sci. 81, 446-452 (2014), arXiv:1305.5973

${ }^{230} \mathrm{C}$. Team, "Crystallography open database," http://www.crystallography.net/cod/, accessed 20 July 2021.

${ }^{231}$ Materials Cloud, "Quantum espresso input generator and structure visualizer," https://www.materialscloud.org/work/tools/qeinputgenerator (), accessed 20 July 2021.

${ }^{232}$ H. J. Monkhorst and J. D. Pack, "Special points for Brillouinzone integrations," Phys. Rev. B 13, 5188-5192 (1976)

${ }^{233}$ M. Cardona, R. K. Kremer, R. Lauck, G. Siegle, A. Muñoz, A. H. Romero, and A. Schindler, "Electronic, vibrational, and thermodynamic properties of $\mathrm{ZnS}$ with zinc-blende and rocksalt structure," Phys. Rev. B 81, 075207 (2010)

${ }^{234}$ Materials Cloud, "Seek-path: the k-path finder and visualizer," https://www.materialscloud.org/work/tools/seekpath (), accessed 20 July 2021.

${ }^{235} \mathrm{~W}$. Setyawan and S. Curtarolo, "High-throughput electronic band structure calculations: Challenges and tools," Comput. Mater. Sci. 49, 299-312 (2010)

${ }^{236}$ A. Jain, S. P. Ong, G. Hautier, W. Chen, W. D. Richards, S. Dacek, S. Cholia, D. Gunter, D. Skinner, G. Ceder, and K. A. Persson, "Commentary: The materials project: A materials genome approach to accelerating materials innovation," APL Mater. 1, 011002 (2013)

${ }^{237}$ T. K. Tran, W. Park, W. Tong, M. M. Kyi, B. K. Wagner, and C. J. Summers, "Photoluminescence properties of $\mathrm{ZnS}$ epilayers," J. Appl. Phys. 81, 2803-2809 (1997)

${ }^{238}$ A. R. McDonald, J. A. Nash, P. S. Nerenberg, K. A. Ball, O. Sode, J. J. Foley, T. L. Windus, and T. D. Crawford, "Building capacity for undergraduate education and training in computational molecular science: A collaboration between the MERCURY consortium and the Molecular Sciences Software Institute," Int. J. Quantum Chem. 120, e26359 (2020) 